\title{
SAMPL7 Challenge Overview: Assessing the reliability of polarizable and non-polarizable methods for host-guest binding free energy calculations
}

5 Martin Amezcua (ORCID: 0000-0002-4926-6542) ${ }^{1}$ and David L. Mobley (ORCID: 0000-0002-1083-5533) (1,2 $^{1}$

- ${ }^{1}$ Department of Pharmaceutical Sciences, University of California, Irvine, Irvine, California 92697, United States;

7 2Department of Chemistry, University of California, Irvine, Irvine, California 92697, United States

$8 \quad$ *For correspondence:

9 dmobley@mobleylab.org (DLM)

Abstract The SAMPL challenges focus on testing and driving progress of computational methods to help guide pharmaceutical drug discovery. However, assessment of methods for predicting binding affinities is often hampered by computational challenges such as conformational sampling, protonation state uncertainties, variation in test sets selected, and even lack of high quality experimental data. SAMPL blind challenges have thus frequently included a component focusing on host-guest binding, which removes some of these challenges while still focusing on molecular recognition. Here, we report on the results of the SAMPL7 blind prediction challenge for host-guest affinity prediction. In this study, we focused on three different host-guest categories - a familiar deep cavity cavitand series which has been featured in several prior challenges (where we examine binding of a series of guests to two hosts), a new series of cyclodextrin derivatives which are monofunctionalized around the rim to add amino acid-like functionality (where we examine binding of a two guests to a series of hosts), and binding of a series of guests to a new acyclic TrimerTrip host which is related to previous cucurbituril hosts. Many predictions used methods based on molecular simulations, and overall success was mixed, though several methods stood out. As in SAMPL6, we find that one strategy for achieving reasonable accuracy here was to make empirical corrections to binding predictions based on previous data for host categories which have been studied well before, though this can be of limited value when new systems are included. Additionally, we found that methods using the AMOEBA polarizable force field had considerable success for the two host categories in which they participated. The new TrimerTrip system was also found to introduce some sampling problems, because multiple conformations may be relevant to binding and interconvert only slowly. Overall, results in this challenge tentatively suggest that further investigation of polarizable force fields for these challenges may be warranted.

\subsection{Keywords}

host-guest binding $\cdot$ free energy $\cdot$ binding affinity $\cdot$ SAMPL $\cdot$ blind challenge $\cdot$ OctaAcid · cyclodextrin · cucurbituril

\subsection{Abbreviations}

SAMPL Statistical Assessment of the Modeling of Proteins and Ligands

AM1-BCC Austin model 1 bond charge correction

RESP Restrained electrostatic potential

REST Replica exchange with solute tempering

FSDAM Fast switching double annihilation method

B2PLYPD3 Beck 2-parameter Lee-Yang-Parr D3 exchange-correlation functional [1] 
B3PW91 Becke 3-parameter Perdew-Wang 91 exchange-correlation functional [2]

GAFF Generalized AMBER force field

CGenFF CHARMM generalized force field

AMOEBA Atomic multipole optimized energetics for biomolecular simulations

DDM Double decoupling method

DFT Density functional theory

QM/MM Mixed quantum mechanics and molecular mechanics

MMPBSA Molecular mechanics Poisson Boltzmann/solvent accessible surface area

MMGBSA Molecular mechanics generalized born/solvent accessible surface area

TIP3P Transferable interaction potential three-point

TIP4PEw Traansferable interaction potential four-point Ewald

OPC3 Optimal 3-point charge

SEM Standard error of the mean

RMSE Root mean squared error

MAE Mean absolute error

ME Mean signed error

$\tau$ Kendall's rank correlation coefficient (Tau)

$\mathbf{R}^{2}$ Coefficient of determination (R-Squared)

QM Quantum Mechanics

MM Molecular Mechanics

\section{Introduction}

Docking and scoring methods have long been used to assist with hit identification and optimization in computer-aided drug design (CADD) [3]. More recently, efforts to improve the reliability of CADD methodologies have gone beyond qualitative docking and scoring towards quantitative modeling [4] via molecular simulations, which can be used to estimate a variety of physical properties of interest $[3,4]$. In this area, predictions of protein-ligand binding free energies have gained much attention for a few decades for their potential to help accelerate small-molecule drug discovery [5], but have received increasing attention recently as this potential begins to be realized $[6,7]$. The long-term goal is to use computational techniques to aid and direct small molecule design to more rapidly and efficiently produce new therapeutics [4]. Right now, much discovery works via a slow cycle of experimental trial and error, but accurate enough free energy methods could dramatically accelerate early stage discovery $[3,5]$.

The accuracy of free energy calculations is dependent on and limited by inaccuracy in the energy model used (i.e., force field used, finite-size effects, and water model) [8], sampling, and the protein-ligand system set up, which can include aspects such as protonation state, chosen tautomer state, and buffer, to name a few [3,5]. Although sources of systematic error in free energy calculations are known, it is difficult to analyze errors when modeling protein-ligand systems due to their flexibility and complexity; such challenges mean that simulations of a few nanoseconds to microseconds may not always adequately sample the relevant conformations of the protein, ligand and environment [3,5]. For this reason, host-guest systems are a great substitute for protein-ligand systems in evaluations of computational methods for predicting free energies of binding [3], as conformational sampling can be less of a challenge.

Host-guest systems are similar to protein-ligand systems in that they also involve binding of a small molecule to a pocket in a receptor, though they have certain differences. We can think of a host as resembling a very small protein molecule (of different chemistry) which has a binding cavity or pocket. A guest is a small molecule which can bind non-covalently to the host. Supramolecular host families such as the cucurbiturils, cavitands, and cyclodextrins have diverse binding affinities and the ability to bind small drug-like compounds with protein-ligand like affinities [4]. Unlike proteins, the hosts are smaller, simpler, and often more rigid [9], removing some of the challenges facing computational modeling of proteins. These characteristics make host-guest systems an ideal substitute to test current computational methodologies used to predict physical properties of interest and investigate issues including binding, receptor flexibility, solvation, hydrogen bonding, the hydrophobic effect, protonation, and tautomers [3]. That is, while prediction of protein-ligand binding is still of interest, host-guest systems can serve to help focus on the accuracy of computational methods themselves, without conflating as many other challenges.

In this work, we describe the recent SAMPL7 host-guest challenge, which allowed participants using diverse methods to 
predict host-guest binding free energies for a variety of guests to three different host families. Here, we give the challenge background, describe the hosts, survey participants' results, and highlight key lessons learned.

\section{SAMPL Challenge Background, History, and Expectations}

\subsection{What is SAMPL? Statistical Assessment of the Modeling of Proteins and Ligands}

The SAMPL (Statistical Assessment of the Modeling of Proteins and Ligands) challenges focus efforts on improving and advancing computational methods through crowdsourcing. Host-guest systems form the basis of one category of the SAMPL challenges (with others focusing on predicting physical properties, and on protein-ligand binding) and typical challenge performance indicates such host-guest systems still pose challenges to contemporary methods [10]. In general, SAMPL challenges provide the community an opportunity to test and compare performance of a variety of computational methods on the same diverse data, an opportunity not always afforded by other datasets. The subsequent release of experimental data allows accuracy to be compared relative to experimental results which were not known when predictions were made, and the subsequent statistical assessment compares methods on equal footing. Thus SAMPL results and the lessons learned are often important for many participants and help drive further methods research.

\subsection{SAMPL fields blind challenges to provide fair tests}

Blind challenges, like SAMPL and companion challenges such the the Drug Design Data Resource (D3R) Grand Challenges, ensure participants do not know experimental values when running calculations [10], ensuring that method comparisons are fair and performance is hopefully indicative of what could be expected in real-world applications to related problems. Occasionally, host(s) or guest(s) are revisited, so related experimental results are available, but we avoid cases where the experimental value being predicted is already available in the literature. A wealth of experimental data is already available, so SAMPL focuses on predictive tests rather than retrospective analysis. The SAMPL challenges are organized in this manner to ensure no participant, even accidentally, adjusts their method to agree with "correct" values thereby introducing bias. For example, when experimental values are known, a naive participant could stop calculations when they agree with the experimental value because they have "converged". Or more subtly, a participant could run calculations with several different sets of settings in the simulation package used and conclude that the settings which gave the best results were optimal, whereas in fact they might be just observing random fluctuations. Blind challenges avoid such opportunities for bias.

SAMPL blind challenges typically involve a host-guest component that provides a platform to fairly evaluate methodologies in computational drug design. Upon evaluation, participants and organizers can assess the lessons learned and the potential value of different methods. Subsequently, computational methods and their algorithms can be calibrated and optimized for application in future blind challenges and in the real world [11].

\subsection{Host-guest systems}

\subsubsection{What are host guest systems?}

As described briefly in Section 1, host-guest systems are similar to protein-ligand systems in that they both involve the binding of a small molecule to a pocket in a receptor. Hosts are mini-receptors, often containing less than 100 non-hydrogen atoms, but are slightly larger than small molecules [9], so the broader field of such chemistry is often called supramolecular chemistry. In host-guest binding, the host binds other small molecules, called guests, in an internal cavity or similar. Usually, hosts don't have large number of possible folds or conformational structures like a protein [9]. Eventually some host-guest systems are well characterized and become part of the driving force behind methodology improvement, with the ultimate goal of transferability to protein-ligand systems [11].

\subsubsection{Why use host guest systems?}

Despite their apparent relative simplicity, host-guest binding has proved a difficult challenge for computation. Large-scale protein-ligand binding free energy studies often report RMS errors in the 1-2 kcal/mol range [6, 7, 12-14], which is considerably better than typical performance in SAMPL host-guest challenges [3, 4, 15-17]. It may be that host-guest systems are "simple" enough that there is essentially nowhere for problems to hide, or confounding factors like polarizability and force field limitations may be more profound in these simple mini-receptors. Alternatively, performance of protein-ligand binding free energy calculations has often been worse in blind challenges like the SAMPL [18] and D3R [19-22] blind challenges than in the large-scale tests cited above, so it may be that typical retrospective tests simply benefit from participants utilizing additional knowledge which is 
not available prospectively or in blind challenges. This is supported to some extent by recent benchmarking work from Merck KGaA [13], and by an earlier industry perspective [23].

\section{Some aspects can pose particular challenges for free energy calculations}

Several different issues arise in the context of binding free energy calculations that can cause particular difficulties or challenges. Here, we survey several major categories of issues which affect some methods participating in SAMPL7.

\subsection{Guests bearing a formal charge can pose methodological challenges}

Molecules with formal charges can pose challenges for molecular simulations, and especially for binding free energy calculations. These challenges, and differences in how they are handled, can be particularly important when studying binding in systems like those considered here.

In general, conducting efficient molecular simulations requires making approximations and simplifications of electrostatic interactions. For example, typically we are interested in bulk or bulk-like behavior, but simulating macroscale systems is cost prohibitive, so we may instead choose to simulate a microscopic box under periodic boundary conditions (PBCs) to minimize edge effects. Alternatively, a modeler might choose to apply effective electrostatic interaction functions.

To effectively treat electrostatic interactions, functions involving cutoff truncation schemes combined with reaction-field (RF) contribution or lattice-summation (LS) methods may be employed [8, 24, 25]. These methods cause the charging component of the calculated free energies to be sensitive to important system parameters like the cutoff radius or the box size [25]. In addition, the raw single-ion solvation free energies from explicit-solvent simulations are extremely sensitive to the boundary conditions and electrostatic interaction treatment [24].

The approximations described above may also introduce bias or offset in the electrostatic potential during the simulation. System-dependent artifacts can also arise from system parameters (such as cutoff radius, box shape and/or size). The artifacts are due to finite-size effects which impact computed charging free energies/binding free energies. While such errors do not have a major effect on computed free energies as long as systems remain net neutral or have a consistent formal charge, they become particularly pronounced when the formal charge of a system changes, such as during an alchemical binding free energy calculation [8] as employed by many SAMPL participants. For this reason methods may need to account and correct for artifacts that may not cancel when a formal charge is alchemically inserted in the system. The sign and magnitude of artifacts depend on the methods used to calculate electrostatic interactions.

The exact sources of such finite-size errors have been described previously. Briefly, the finite-size error in ligand/guest charging (and by extension, binding) free energies originates from at least four different physical effects in periodic systems: (1) Periodicity-induced net-charge interactions; (2) Periodicity-induced net charge undersolvation; (3) Discrete solvent effects; and (4) Residual integrated potential effects [8].

There have been some attempts to address these issues; particularly, both instantaneous and post-simulation correction strategies have been proposed $[8,24,26]$. One approach is to apply various after-the-fact corrections to computed free energies $[8,24,26]$. Alternatively, others have proposed applying a correction strategy during simulations, which has been called a co-alchemical ion approach, wherein an alchemical perturbation of a charged moiety is simultaneously performed with a counter-alchemical charge perturbation of a remote molecule (i.e. a counter-ion) [8, 25]. In other words, in this approach, the system is maintained net neutral by offsetting a charge change in one portion of a system with a compensating change in another portion of the system. The goal in this approach is to ensure that errors from finite-size effects are negligible. Post simulation strategies include charge-correction terms which have been shown to work for LS and RF, and can be evaluated via numerical and analytical methods $[8,24,27,28]$.

\subsection{Polarization can potentially pose particular challenges}

Charged molecules - like those frequent in SAMPL7 - can also pose particular challenges because of strong electrostatic interactions with their immediate surroundings. This poses two challenges which are particularly relevant here - first, any polarization of the surroundings may be particularly important. Second, other electrostatic interactions are quite strong, including interactions with surrounding ions. These can include screening effects, but also relatively more specific interactions.

Polarization is a phenomenon where atoms and molecules induce changes in the electron distributions of other atoms/molecules they interact with [29]. This effect grows stronger/more pronounced the stronger the electrostatic interactions and/or the more polarizable the atoms involved. Because of their strong electrostatics, then, the electrostatic interactions of charged groups 
can be particularly affected by polarization. Additionally, anions such as iodide and bromide are highly polarizable, including anions with phosphate or sulfate moieties which are present in a wide range of biomolecules [30, 31]. Phosphates and sulfates play important roles in biological functions, interactions, and are present in drug-like molecules [31]. On the other hand, small cations have low polarizability but can still strongly polarize their environment when it is polarizable.

Much molecular modeling uses classical fixed-charge force fields without an explicit accounting for polarization [31]. Such two-body additive force fields are implicitly polarized to hopefully match a level of polarization appropriate on average for condensed-phase simulations [32-34]. This is true for common force fields in the AMBER, CHARMM, GROMOS and OPLS families (e.g. GAFF [35, 36], OpenFF [37], CGenFF [38-40], and OPLS [41, 42]); these neglect polarization for computational efficiency. It's possible that the approximations made by these fixed-charge force fields may result in particularly large errors in systems like those examined here [43].

Polarizability may also be particularly important for these systems due to the water model. Particularly, with fixed-charge force fields, the water model is also non-polarizable, which may be an especially bad approximation for systems like these where water interactions with a buried hydrophobic cavity are at play [43]. The expectation is that binding in host-guest systems like those examined here are heavily influenced by the hydrophobic effect, and the hydrophobic effect will certainly be strongly influenced by properties like polarizability.

Fixed point charge water models are limited in some ways by their use of the same partial charges to empirically fit the potential energy landscape and dipole moment, two distinct water properties [44, 45]. Inevitably, the choice in water model (many listed in [46, 47]) may also dictate the accuracy in (a) solvation, (b) dielectric constant, and (c) dipole moment [44], and affect ionic behavior along with many other properties. Previous work in the Gilson lab indicated that even fixed-charge water models can vary dramatically in water placement and orientation around hosts as well as in thermodynamic properties like the enthalpy of binding $[48,49]$, and it seems likely that polarizable models may exhibit even larger differences.

Polarizable force fields potentially help address some of these concerns and challenges. PIPF (polarizable intermolecular potential function) and AMOEBA were among the first polarizable force fields developed, and have been in development since the 1990s [46]. Polarizable force fields, and their importance for such systems, are explained in Section 2. In addition, popular general force fields such as AMBER, OPLS, GROMOS, and CHARMM are continuously evolving and polarizable versions of some of these are available [46]. One example of the latter is a recent release of CHARMM's balanced Drude polarizable force field [31]. However, polarizable force fields have been applied relatively seldom in SAMPL challenges; AMOEBA was used in some prior host-guest challenges [11], but the Drude polarizable force field has yet to be used in a SAMPL challenge.

In other words, polarizable force fields add additional complexity to the physical model used in describing these systems, potentially providing additional accuracy but with additional computational cost. However, for some host-guest systems, this may be particularly important for several physical reasons. First, these systems often exhibit strong electrostatic interactions in a buried, relatively hydrophobic environment, meaning that the precise degree of polarization and environmental shielding may be a key determinant. Polarizability may affect the strength of charge-charge interactions, and may strongly modulate the shielding effect of the environment. Additionally, the hydrophobic effect can be a key determinant of binding, and this is also likely strongly modulated by polarization of the water and host.

Polarizable force fields have shown some promise in prior SAMPL challenges. In the SAMPL6 host-guest challenge, a method using the AMOEBA force field was employed on CB8 with 14 guests ranging from small organic molecules to larger drug-like compounds, including approved drugs. The initial results had an ME and RMSE of 2.63 and $3.62 \mathrm{kcal} / \mathrm{mol}$ respectively, and interestingly, this method was able to correctly identify questionable host-guest complex ratios of CB8 with guests 11 and 12 [11]. The correct respective ratios for these systems were 1:1 and 1:2, and these were a bonus challenge in SAMPL6. It was found that there was significant overestimation of guests 2 and 3 (Palonosetron and Quinine) and were presumed to be due to (a) AMOEBA parameters for the host resulted in single and/or double indentation of the macrocycle and (b) conformers of flexible guests locked during solvation in water vs binding in solvated complex [11]. In their subsequent studies, revised AMOEBA results reported improved ME and RMSE of 1.20 and $1.68 \mathrm{kcal} / \mathrm{mol}$ respectively, though this was after challenge results were released. In total 8 of the 15 predicted free energies were within $0.65 \mathrm{kcal} / \mathrm{mol}$ of experiment while the predictions for Palonosetron and Quinine guests were in better agreement with experiments. The improvements were attributed to two factors: (a) the value of key torsion parameters for $\mathrm{C}(\mathrm{N})$ - $\mathrm{C}$-amide $\mathrm{N}$-carbonyl carbons of $\mathrm{CB} 8$ and $\mathrm{CB} 7$ were adjusted to improve the flexibility description of the host ring system and (b) a double annihilation scheme of electrostatics and van der Waals with annihilation of key guest torsions yielded much better conformational sampling and hence predictive accuracy. However, through the SAMPL6 challenge we had yet seen AMOEBA dramatically outperform other methods prospectively. 


\subsection{Differences in system treatment could also be important in some cases}

Empirical force fields' predictive power can be limited by the quality of their parameters and the parameterization. Parameters are not always available for all relevant chemistry, or may not be of equal quality for all chemistry of interest. For example, experiments for all components of the SAMPL7 host-guest challenge were done in sodium phosphate buffer (of varying concentration and $\mathrm{pH}$ ). However, because of concerns about the quality of phosphate force field parameters, we conducted our reference calculations in sodium chloride (of the same ionic strength) instead. While this choice seems reasonable and is not uncommon in molecular modeling, it might affect computed free energies.

Particularly, the type of salt and its concentration can alter the solubility of a solute (e.g. in what is known as the Hofmeister effect) $[50,51]$. Such salt dependence also interacts with the choice of water model. Particularly, one computational study reported surprising differences in the salt dependency of binding enthalpy (comparing TIP3P, SPC/E, TIP4P-EW, and OPC water models) during MD simulations for cucurbit[7]uril host with a neutral guest [52]. Despite the system being non-ionized, the salt concentration (and the choice of sodium and chloride parameters) affected the behavior and thermodynamics of water, raising issues regarding selection and adjustment of water models for charged groups [52]. Incorrect ionic behavior (i.e. dielectric constant, dipole moment, solvation, and excessive ion-pairing and/or ion pairing strength) has been shown to be due to unbalanced force field parameters [31, 44].

In the present SAMPL challenge, some participants did not use any ions beyond counter ions to neutralize their systems. However, salt concentration is known to play a significant role in modulating host-guest binding affinities experimentally in some cases $[9,53]$. Thus, if salt concentration proves important here, such differences in protocol could produce a systematic difference between methods.

\subsection{Some methods require considerable expertise to use successfully}

Some methods for binding prediction require extensive knowledge and expertise. For example, a person with little computational experience may not be able to conduct a successful free energy calculation given the historical difficulty of setting up such simulations. Few available software tools are user-friendly enough that one might be able to simply insert receptor and ligand files and obtain an accurate estimate of a property like a binding free energy. This likely affects accuracy; it's conceivable that users providing the same input files to the same package could obtain dramatically different results because of different choices of protocol.

Some tools provide a relatively straightforward interface for free energy calculations, at least, like YANK, but even YANK still requires a command-line interface and a wide variety of settings can affect computed values. Other tools like those from Schrödinger and the Chemical Computing Group allow free energy calculations from GUI (Graphical User Interface, and the Schrödinger tools remove many key choices from the hands of users. However, we are not yet aware of a successful application of these tools to host-guest binding.

\subsection{Here, we avoid multimeric systems which introduce additional complications}

Binding which involves stoichiometries other than 1:1 can be considered multimeric association. Some proteins exhibit this behavior, where a single protein molecule co-assembles with other proteins to form a complex; in other cases, a protein might oligomerize only on binding of a ligand or ligands. The reverse can also happen, with multiple ligands binding to a single protein, etc. The same holds true for some host-guest systems, with these systems exhibiting binding that is not 1:1 [54, 55], complicating both experimental measurement of binding and computational prediction thereof. Here, we worked with experimental collaborators to deliberately ensure the challenge focuses on systems exhibiting 1:1 binding. However, the formation of host-guest multimeric complexes can even depend on the guest identity [55].

With multimeric host-guest complexes, cooperativity may play a role. Cooperativity occurs when a binding event can either increase or decrease the strength of subsequent binding events [56]. In the presence of ions, electrostatic attractions can also lead to cooperativity [57]. Indeed, experiments must verify 1:1 binding (as was done here) otherwise computation would need to consider other possibilities.

\section{Previous SAMPL host-guest challenges used similar hosts}

Previously SAMPL challenges have included a variety of host-guest systems, but the majority of SAMPL hosts have been in the cucurbituril [58] and Gibb deep cavity cavitand (often "OctaAcid") families [59] thanks to the contributions of Lyle Isaacs and 
Bruce Gibb's labs. There have been several analogs of these two families since host-guest systems first appeared in SAMPL3. SAMPL7 includes several analogs in the cyclodextrin [60] family thanks to Michael Gilson's lab.

Study of these various systems, in SAMPL and elsewhere, can help provide insight into the particular challenges each system presents. However, conclusions are not always clear; sometimes, performance remains highly variable across several challenges.

Particularly, performance in prior SAMPL challenges was highly variable by method and target, and no clear method emerged as reliable across all systems or most systems. Both SAMPL3 and SAMPL4 included some guests in cucurbituril family [15, 6164], with the best RMS errors typically being around $2.5 \mathrm{kcal} / \mathrm{mol}$ unless empirical corrections were included [61, 65], and no method stood out across both challenges [17]. SAMPL4 also included cavitands. In SAMPL5, the best RMS error was closer to 3 $\mathrm{kcal} / \mathrm{mol}$ [61], but correlation with experiment for this approach was not good. Methods based on explicit solvent and electronic structure calculations were noted to appear relatively consistent and generally provide the greatest reliability across all SAMPL challenges [66], but also had considerable room for improvement. In general, predictions for cavitands seemed to be modestly more accurate whereas clip-based hosts have been more challenging in prior challenges (like CBClip in SAMPL5 [66]). Thus, in the present challenge, we hoped to learn whether we might see a method or methods with significantly improved accuracy relative to prior challenges, and whether one might emerge that performs reasonably well (e.g. RMS error under $3 \mathrm{kcal} / \mathrm{mol}$ ) across multiple host classes, as this has not typically been the case in prior challenges.

\section{SAMPL7 Host-Guest Systems and Challenge Organization}

The SAMPL7 host-guest challenge involved three different systems or categories which we explain here - one focusing on cucurbituril-derivatives, one focusing on Gibb deep cavity cavitands (GDCCs), and one focusing on modified cyclodextrins.

\subsection{Cucurbiturils and derivatives (CB[n], CB-Clip and TrimerTrip)}

Cucubiturils are a common and relatively well-studied system for host-guest binding [9] which have been featured in some prior SAMPL challenges.

Many cucurbiturils ( $\mathrm{CB}[\mathrm{n}] \mathrm{s}$ ) have been synthesized by the Isaacs Lab, and several featured in previous SAMPL challenges. The potential applications of cucurbiturils include use as solublizing excipients for insoluble drugs, sequestrants for drugs of abuse and neuromuscular blockers, and pH triggered delivery agents [58]. This family of hosts typically have a molecular structure containing $n$ glycoluril units connected via $2 n$ methylene bridges, forming a barrel shaped macrocycle with a central hydrophobic cavity. In addition, cucurbiturils contain electrostatic carbonyls protruding out from the hydrophobic cavity.

In the SAMPL7 challenge, the host is not a classic cucurbituril, as instead of being a macrocycle, it is a clip-shaped molecule based on similar chemistry. Particularly, the host is an acyclic cucurbituril clip composed of a glycoluril trimer capped with aromatic triptycene sidewalls at both ends (here called TrimerTrip, as it is a trimer of glycoluril units with triptycenes), and four sulfonate solubilizing groups protruding out from the sidewalls (Figure 1) [58]. The sulfonate groups also enhance ion-ion interactions with cationic guests [67], which are typical cucurbituril binders. Acyclic CB[n]-type receptors often take on a C-shape due to their increase in flexibility $[58,67,68]$. Experimentalists synthesized acyclic cucurbiturils with the idea to help increase the binding strength and capacity for different guests, including macrocyclic guests.

Typically, CB[n]-guest complexes have very high affinity, especially for charged hydrophobic ammonium guests similar to those of the SAMPL7 challenge (Figure 1). This high affinity is due to the presence of intracavity waters lacking a full complement of hydrogen bonds. The lack of hydrogen bonds is known to provide an enthalpic driving force for binding to macrocyclic CB[n] complexes [69]. In terms of CB[n]-guest complex interactions, the charged nitrogen group on guests interacts with oxygens from the carbonyl portal of the host. The latter contributes to limiting the number of poses that need to be considered [11], at least in cyclic hosts.

CB7 was used as a basis for host-guest benchmarking (including on binding of guests with adamantane and aromatic ring cores) since some of its properties and characteristics made it a convenient host both computationally and experimentally [9]. Four insights and challenges for CB7 are described [9] and some may be transferable to a clip type cucurbituril. (1) The tight exit portal of CB7 makes it difficult for guests with bulky hydrophobic cores such as adamantyl to fit through the portal and hence lead to convergence problems. (2) The timescales of wetting and dewetting events may be large compared to typical simulation timescales. In CB7, when gradually decoupling a guest there is a large fluctuation of waters in the host cavity. The latter occurs when the guest is partially decoupled and may also lead to convergence problems. (3) Experimental and computational binding thermodynamics are sensitive to the salt composition and concentration (for buffer conditions). (4) Guests with formal charges can pose challenges for binding free energy calculations (Section 3.1). 
g1<smiles>[NH3+]CCCC[NH3+]</smiles>

g2<smiles>[NH3+]CCCCC[NH3+]</smiles>

g3<smiles></smiles>

g12<smiles>CCCCCC[N+](C)(C)C</smiles>

g6<smiles>[NH]CCCCCCCC[NH3+]</smiles>

q16<smiles>[NH3+]CCCCCCCCCC[NH3+]</smiles>

g17
Previous studies of cucurbiturils, including CB7, have highlighted the importance of host and guest sampling, salt effects, and water model. Sampling of the CB7 host is thought to be straightforward because it is fairly rigid. However, guest binding modes might be challenging to adequately sample, especially for the more flexible guests. In the presence of buffer and/or

\section{$\mathrm{R}=\left(\mathrm{CH}_{2}\right)_{3} \mathrm{SO}_{3} \mathrm{Na}$}

g8

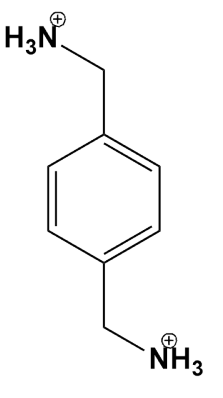

g7<smiles>[NH3+][C@H]1CC[C@@H]([NH3+])CC1</smiles>

g18<smiles>C[n+]1ccc(-c2cc[n+](C)cc2)cc1</smiles>

g19<smiles>C[N+]1(C)CCC(C2CC[N+](C)(C)CC2)CC1</smiles><smiles>[NH3+]C12CC3CC(CC(C3)C1)C2</smiles>

g9

g6

Figure 1. Structures of the TrimerTrip host and guest molecules for the SAMPL7 Host-Guest Blind Challenge. The acyclic CB[n]-type receptor, TrimerTrip, is shown on the top. It is composed of a glycoluril trimer with aromatic triptycene sidewalls at both ends, and four sulfonate groups to increase its solubility. The host takes on a C-shape and binds guests inside the cavity. The guests for the SAMPL7 challenge have the characteristics of typical CB[n] binders. The guests are grouped here, with the aliphatic chains on the left, and the cyclic and aromatics on the right. 
salt, ions may compete with guests for the binding site. In addition, cationic guests could have interactions with counter-ions in solution, lowering affinity compared to zero-salt conditions [9]. One previous study showed a $6.4-6.8 \mathrm{kcal} / \mathrm{mol}$ dependence on salt concentration $[9,70]$. The water structure around CB7 is sensitive to the choice of water model, and water is important in modulating binding in SAMPL7 systems. The choice of water model is also likely to have an impact on the number of sodium ions that must be displaced upon host-guest binding.

While these insights result from studies on CB7, some of them may carry over to the TrimerTrip host studied here. However, unlike its macrocyclic derivatives, TrimerTrip is acyclic and able to flex the methylene bridged glycoluril trimer backbone [68]. Hence, with more degrees of flexibility sampling of TrimerTrip may not be as straightforward. TrimerTrip, like the Calabadion "cousins" in the family of cucurbiturils, may allow guest cationic groups to interact with other regions of the host rather than the carbonyl portals as in $\mathrm{CB}[\mathrm{n}]$ macrocycles [68], which may complicate guest sampling.

Previous acyclic CB[n]-type receptors contain a central glycoluril oligomer (monomer, dimer, and tetramer) with aromatic triptycene sidewalls, just like TrimerTrip. These clip like receptors retain the essential molecular recognition properties of macrocyclic CB[n] [71]. The monomer [67], dimer [71], and tetramer [62, 71] clips are able to encapsulate typical hydrophobic cationic guests which also bind to macrocyclic $\mathrm{CB}[\mathrm{n}] \mathrm{s}$. In addition, the dimer and tetramer display similar host-guest properties [71]. While TrimerTrip is a distinct host, it shares substantial similarity with these previous receptors and we expect it to exhibit relatively similar behaviors in binding to guests.

\subsection{Gibb Deep Cavity Cavitands (GDCCs) - OctaAcid (OA) and exo-OctaAcid (exo-OA)}

Of the several members in the GDCC host family [59], two have been used in several previous SAMPL challenges thanks to the Gibb group's participation. Those featured in previous SAMPL challenges include OctaAcid (OA) and tetra-endomethyl OctaAcid (TEMOA). A newer exo-OctaAcid (exo-OA) along with OA are part of the SAMPL7 host-guest blind challenge (Figure 2). The guests for this system are diverse in their size and bulkiness, but typically have either a carboxylate or quaternary ammonium (Figure 2).

$\mathrm{OA}$ and exo-OA have a deep and hydrophobic basket-shaped pocket, and are fairly rigid $[9,54]$. In total there are eight carboxylate groups in both OA and exo-OA. The proprionate groups at the exterior site of the cavity are the same in both hosts. The difference between the two hosts is the location of 4 carboxylates around the cavity opening. For OA the carboxylates are protruding out of the cavity while for exo-OA they are at the cavity entrance (Figure 2).

GDCCs have been used in SAMPL3-7 and there is much experimental data $[9,43,59,72]$ and insight available. This family of hosts bind guests with a hydrophobic moiety that fits the pocket and a hydrophilic group which points out towards the solvent [9].

The GDCCs have been shown to bind diverse guests varying in polarity, positively and negatively charged, as well as organic cations and anions $[9,73,74]$. The latter has been shown for OA, where binding thermodynamics is sensitive to the concentration and type of anions present. Shifts in binding enthalpies and free energies of approximately $10 \mathrm{kcal} / \mathrm{mol}$ and $2 \mathrm{kcal} / \mathrm{mol}$ respectively [50] have been observed and attributed to the competition between guests and anions leading to entropy-enthalpy trade-offs $[9,50]$. In addition, experimental and computational simulation results show that de-wetting of GDCCs leads to increased guest affinity, because water cannot compete for the pocket [59, 72].

In the presence of elongated guests, such as a long aliphatic chain, two OA hosts can encapsulate a guest forming a ternary complex. This phenomena is more likely to occur as polarity decreases for the groups at both ends of the guest [73]. However, as described earlier in section 3.5, SAMPL7 was designed around systems which exhibit 1:1 binding. Isothermal titration calorimetry (ITC) experiments have shown that short-chain fatty acids, amphiphilic molecules, and large polarizable anions form 1:1 complexes [72], as do the guests reported here.

Previous work has proposed benchmarking free energy calculations on host-guest systems; for GDCCs, the proposed benchmark included OA binding to guests with adamantane, aromatic, and saturated cyclic carboxylates. These host-guest systems were chosen because of the broad range of binding free energy values produced, and because both host and guests are small and rigid enough to confidently converge binding free energy calculations [9]. Several key challenges were highlighted by prior work: (1) a tight entry/exit portal may create a barrier and prevent entry or exit of guests with bulky hydrophobic cores. Hence, this can hinder sampling of guests leading to convergence problems. (2) It is important to ensure adequate host conformational sampling (though the motions may be slow), particularly of the propionoic acid groups. Benzoic acid flips (at the rim of the cavity) have also been reported from several simulations $[3,9,61]$, though these have not been verified experimentally and may be irrelevant to binding thermodynamics. However, it has been noted that the benzoic acid flips might be an important challenge in some force fields. (3) Waters move only slowly into and out of the cavity, with the number fluctuating over tens of nanoseconds $[9,75]$. (4) Salt concentration and buffer conditions may modulate binding to GDCCs. Additionally, (5) charged guests may introduce finite-size artifacts. (6) Strong electrostatic interactions could result in modified protonation states of the 

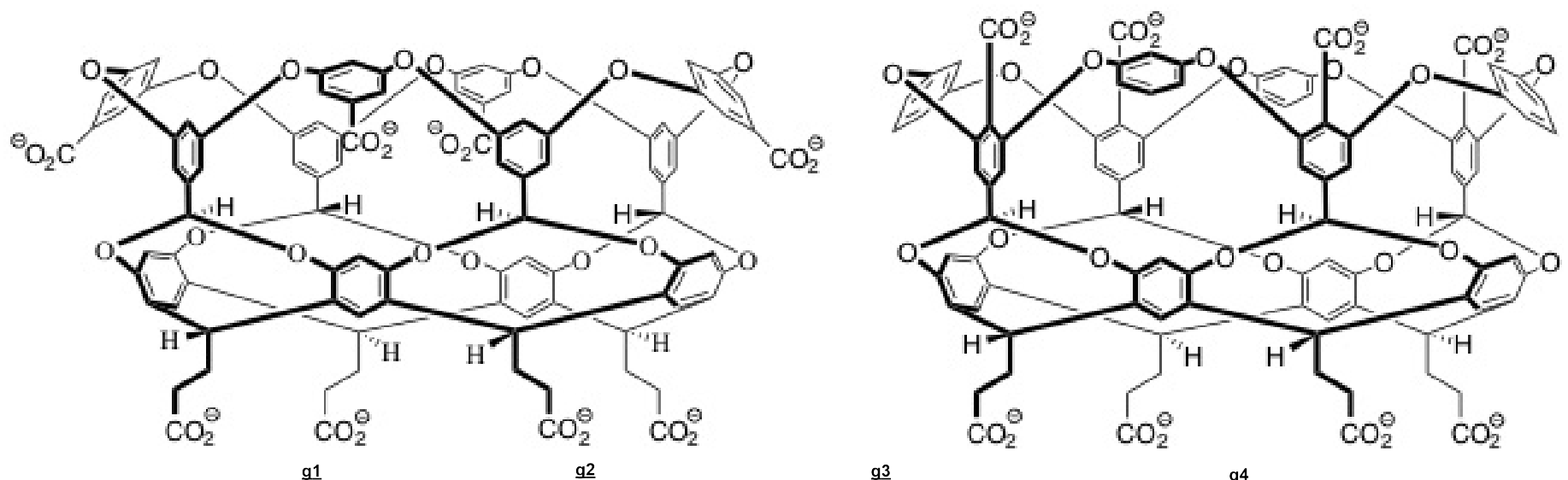

g3
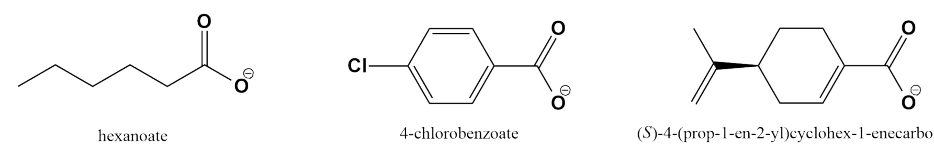

(S)-4-(prop-1-en-2-yl)cyclohex-1-enecarboxylate

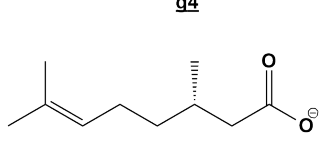

(S)-3,7-dimethyloct-6-enoate

g5

q6

g7
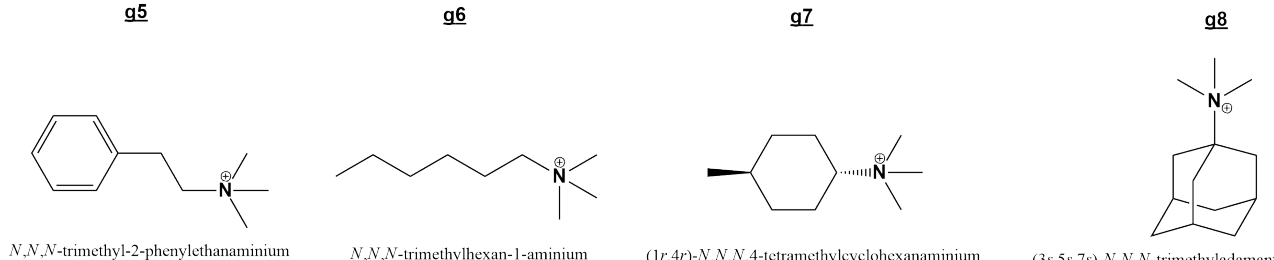

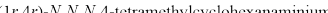

$(3 s, 5 s, 7 s)-N N N$-trimethyladamantan-1-aminium

Figure 2. Structures of the GDCC host and guest molecules for the SAMPL7 Host-Guest Blind Challenge. (top left) OctaAcid, (top right) exo-OctaAcid; (bottom) guests. The difference between both of the hosts is the placement of the carboxylate groups near the cavity opening. While the carboxylates protrude outward away from the cavity in OA, in exoOA they are at the rim of the cavity opening. The guests for SAMPL7 are named g1 - g8, with four guests with a carboxylate group, and four with a quaternary ammonium group. For the OA host, guests g1 - g6 have binding free energies which were previously reported and thus calculation of values was made optional for participants.

host and/or guest. Acidic guests could be protonated, or two of the propionate groups could retain an acidic proton because they are in close proximity and can hydrogen bond. At the rim of the cavity a guest may also modulate protonation state of the neighboring carboxylates.

\subsection{Cyclodextrins (CDs) and cyclodextrin derivatives}

Cyclodextrin (CD) family hosts are composed of chiral glucose monomers linked to yield a cyclic polymer. The SAMPL7 challenge focused on modified CDs provided by the Gilson lab, which synthesized monofunctionalized derivatives differing by addition of a substituent projecting outward from a primary or secondary face hydroxyl of the cyclic oligosaccharide (Figure 3). The CD host derivatives are aimed to have a truncated cone shape (Figure 4) with a hydrophobic cavity and a hydrophilic surface, while the substituents are intended to alter the host's chemical and physical properties. The new host substituents introduce new host-guest interactions, while retaining some of the same binding characteristics [76].

While typical SAMPL host-guest challenges have focused on binding of a series of guests to one or two hosts, one unique aspect of this portion of the challenge is that it focuses on binding of just two guests to a series of related hosts.

Previous studies on CDs ( $\alpha$ - CD, $\beta$-CD, and mono-3-carboxyproponamido- $\beta C D)$ report two distinct bound states for each hostguest pair. The first bound state, called the "primary orientation", has the guest polar group (i.e., alcohol, ammonium, carboxylate) towards the glucose subunits primary alcohols, while the "secondary orientation" has the guest polar group towards the secondary alcohol [76, 77] (Figure 4). Though a possible third "surface orientation"/binding mode has been speculated to exist, it may be this is a transition needed for the guest to flip from primary to secondary phase orientation or vice-versa [43]. The difference in binding free energy for the two main orientations has been reported as being about $2 \mathrm{kcal} / \mathrm{mol}$ and up to $5 \mathrm{kcal} / \mathrm{mol}$ using several different force fields [77], with this of course also depending on the guest. The same report suggested that using 


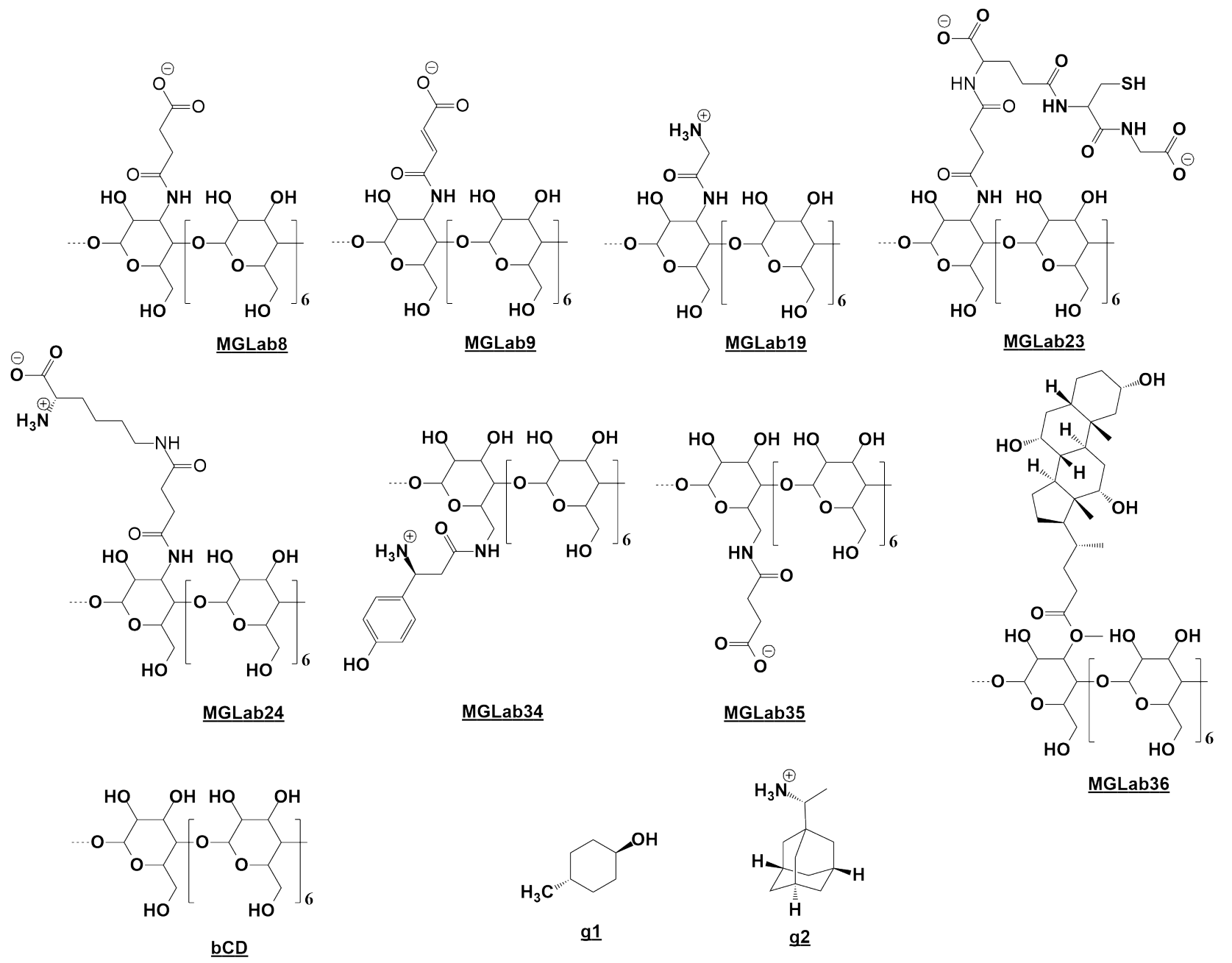

Figure 3. Structures of the Cyclodextrin host derivatives and guests for the SAMPL7 Host-Guest Blind Challenge. Cyclodextrin derivatives are a series of macrocycles composed of seven glucose subunits linked by 1,4 glycosidic bonds. The native $\beta$-Cyclodextrin (bCD) contains the primary $\left(2^{\prime} \mathrm{OH}\right)$ and secondary glucose subunit hydroxyls, while all of the cyclodextrin derivatives (MGLab\#) differ by a substituent at either of these positions. MGLab8, MGLab9, MGLab19, MGLab23, MGLab24, and MGLab36 have substituents out from the top or primary face (wide opening), while MGLab34 and MGLab35 have the substituents out from the bottom or secondary face (narrow opening). The two guests are trans-4-methylcyclohexanol (g1) and cationic R-Rimantadine (g2).

GAFF v2.1 better models the flexibility of $\beta$-CD compared to the SMIRNOOFF99Frosst and GAFF v1.7 force fields also examined.

The guests studied here have been reported to bind native $\beta$-CD, mono-3-carboxyproponamido- $\beta$-CD, and $\beta$-CD substituted with an amine at the 3 position (secondary face). Rimantadine (Figure 3) binds beta-CD and mono-3-carboxyproponamido- $\beta C D$ with its cationic ammonium group projecting out from the secondary face $[76,78]$. On the other hand rimantadine prefers the primary orientation when binding $\beta$-CD with an amine at the 3 position. Both 4-methyl-cyclohexanol (g1) and rimantadine (g2) (Figure 3) may bind to the new $\beta$-CD derivative hosts (MGLab9 through MGLab36 Figure 3 ) in either of the three orientations. However, it was hypothesized that the rimantadine head group would be oriented towards a negatively charged substituent and away from a positively charged one [43].

\subsection{Challenge Organization and Format}

The SAMPL7 host-guest blind challenge was organized so participants may submit a ranked submission, a non-ranked submission, or both for any or all of the three host-guest systems. Participants were advised to submit their best method as their ranked submission since only one ranked submission is allowed, as detailed below.

Participants were provided with pre-prepared host and guest structures, with SMILES strings, mol2, PDB and sdf files provided for all compounds. We made an effort to provide reasonable protonation states, etc., but also provided disclaimers that 

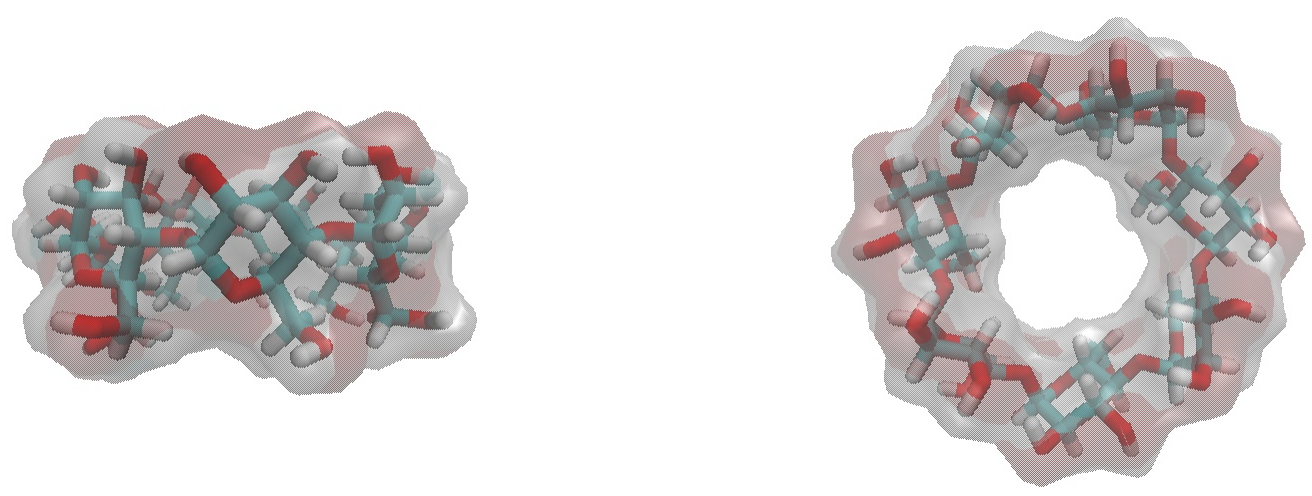

Figure 4. $\beta C D$ host structures describing primary and secondary guest binding orientations. $\beta C D$ and its derivatives are known to bind guests in two orientations, primary and secondary. The primary binding orientation is when an asymmetric guests polar head group projects out towards the glucose primary alcohol or the larger opening (up). The secondary binding orientation is when a guests polar head group projects towards the secondary alcohol or the smaller opening (down).

participants should carefully consider the choice of protonation state, etc. All provided data/instructions are available in the SAMPL7 GitHub repository (https://github.com/samplchallenges/SAMPL7).

Participant submissions followed a prescribed template and included predicted values and uncertainties, as well as method and participant information and other details, All submission files are available in the GitHub repository. Predicted values were optionally allowed to include binding enthalpy.

Only ranked submissions were considered in challenge analysis. Groups were able to submit multiple submissions, but needed to designate additional submissions as non-ranked. Non-ranked submissions, or additional submissions, allow "benchmarking" of methods. For example, for a particular method a participant can change one parameter in their methodology (i.e. charging method, host conformer, guest pose, water model, etc.) to ascertain its impact on predictions. In previous challenges, participants were allowed multiple ranked submissions; the shift to a single ranked submission per participant is new to SAMPL7. This change was made to reduce the potential for multiple shots on goal to be more fair to groups which only submit one set of predictions.

In addition to the formal predictions, one member of our team (MA) conducted a set of blind reference calculations which were submitted informally in the non-ranked category. Data collection for TrimerTrip and its 16 guests (Figure 1) of this challenge was completed around August of 2019 and a challenge submission deadline of October 4, 2019 was set to avoid delaying the experimental publication. The GDCC dataset was finalized on May 25, 2019 and its submission deadline, along with that for the Cyclodextrin derivative challenge, was set to November 4, 2019. Submissions for OA with g1-g6 (Figure 2) guests were optional (and not part of rankings) since these have been reported in previous challenges and literature values are available. In addition, submitting binding enthalpies for GDCC predictions were optional. Similarly, for the Cyclodextrin derivatives dataset, predictions for g1 and g2 binding to $\beta$-cyclodextrin (Figure 3) were optional since literature values for these compounds are available.

As noted above, we provided input files in a variety of formats. Participants were advised that (a) further equilibration of the host with the guest might or might not be needed (for TrimerTrip, we pre-equilibrated the host structure as discussed in Methods) and (b) to exercise their best judgment on the state modeled (i.e protonation, conformer, binding mode, etc.). In essence, part of the host-guest challenge for some systems included binding mode prediction.

\section{Methods}

In this section we describe the details of our own reference calculations, give a general overview of methods used by participants' submissions, summarize key experimental details and methodology (the experimental studies will be published elsewhere [59, 
60, 68]), and describe our statistical analysis and evaluation approach.

\subsection{Absolute Binding Free Energy Predictions}

\subsubsection{Reference Calculation Methodology}

In this section we give details of our own reference calculations. These reference calculations were informally part of the challenge and used as additional methods for comparison. These calculations were also conducted blindly and were informally submitted as a "non-ranked" category, as they do not constitute a formal part of the challenge but are provided as a point of comparison.

Reference calculations were done using an alchemical free energy calculation toolkit known as YANK [10, 79]. YANK provides several schemes for sampling from multiple thermodynamic states. For reference calculations we applied the replica exchange sampler (also known as Hamiltonian Exchange) [79, 80], using the OpenMM simulation engine [81-85]. Free energies are estimated using the multistate Bennet acceptance-ratio (MBAR) [86]. (For details on the thermodynamic cycle used in YANK and the theory see http://getyank.org/latest/theory.html)

Initially, test simulations were done with the goal to determine if we could identify and apply a reasonable single protocol to run all host-guest systems. However, due to the guest formal charges and the diversity of the hosts and guests we guessed that successful protocols (especially lambda spacings) would be system dependent. For the simulations, harmonic distance restraints were used to allow the guest to explore the cavity and different modes since the binding mode of some guests were unknown. We ended up choosing two reasonable protocols, varying in number of lambda windows (with all other simulation parameters kept consistent), with one being for systems with neutral guests and a second for guests with a formal charge. The protocol for neutral guests had 31 lambda windows and was based on a previous protocol used on $\beta$-CD with cyclopentanol as the guest. This protocol was tested on $\beta$-CD with 4-methyl-cyclohexanol as the guest. For systems with a charged guest, we ran a test free energy calculation using YANK's automatic pipeline to determine the best alchemical path (lambda windows and values) based on a $\beta$-CD and the positively charged rimantadine (g2) guest, resulting in 61 lambda windows. Both of the test calculations were within $4 \mathrm{kcal} / \mathrm{mol}$ of experimental values [76] upon completion, and simulation health reports showed reasonable exchange between replicas and exhibited apparently reasonable convergence. However, in the case of the charged guest, convergence was not as convincing at similar time scales. For example, the test calculation for the neutral guest showed reasonable convergence by 14 ns per window while in the case of a charged guest, simulations were run for 26 ns per window and convergence was still not as obvious.

The "neutral guest" protocol described above (31 lambda windows) was used to run all simulations in the cyclodextrin dataset with guest g1, for 16 ns per lambda window when free energy estimates appeared converged. On the other hand, the "charged guest" protocol (61 lambda windows) was used for the remaining host-guest systems across all datasets since all other guests bore a formal charge. In this case simulations, were run until free estimates apparently converged or up to $30 \mathrm{~ns}$ per lambda window, which ever came first. First, to determine feasible cross application of the "charged guest" protocol to different systems (GDCC and TrimerTrip datasets), the charged protocol was tested on OA-g2 and clip-g11. Experimental data for OA-g2 was available from a previous SAMPL challenge, so this was an ideal system to test the protocol. The OA-g2 test resulted in predicted free energy within $4 \mathrm{kcal} / \mathrm{mol}$, after running the simulation to $26 \mathrm{~ns}$ per window. A health report for the OA-g2 simulation showed reasonable mixing between replicas, and there was apparent convergence. However sampling of replicas in individual states was not ideal. For the clip-g11 test simulation (for TrimerTrip dataset), the protocol was initially deemed reasonable based on YANK's health report (with mixing_cutoff and mixing_warning_threshold options at default 0.05 and 0.9 settings, respectively) which can detect insufficient replica mixing or number of swaps between states and thus issue warnings. Warning messages were not issued in this test case. However, in this test case sampling of replicas in individual states was not ideal and the calculations apparently did not fully converge even after $30 \mathrm{~ns}$ per window. For this reason all simulations for TrimerTrip were run for $30 \mathrm{~ns}$ per window in an attempt to obtain reasonable convergence, though after the fact convergence was only apparent for clip-g1 of TrimerTrip dataset. In addition, an "open" host conformer was extracted from the clip-g11 test simulation trajectory, the guest was docked to the open host conformer, and simulation (found in Docking/GAFF/YANK_REF_2) was re-run in an attempt to allow the host to relax and adapt to the bulky guest. Still longer simulations, or protocol optimizations, might be needed for better converged results.

Reference calculations were conducted using GAFF parameters and AM1-BCC charges. GAFF parameters and guest AM1BCC charges were assigned using Antechamber, and AM1-BCC charges for the host were assigned using the OpenEye toolkits because Antechamber could not charge the hosts. The starting poses were determined by docking via AutoDock/Vina and the 
top scoring pose was selected. A host-guest complex was manually created in tLeap and TIP3P was used to solvate the host-guest complex and the guest. In addition sodium and chloride were manually added as counter ions, and additional ions were added to mimic experimental buffer conditions. Subsequently AMBER restart, topology, and input coordinate files were generated with tLeap. The starting simulation files (AMBER restart/coordinate (rst7) and topology (prmtop)), workflow and methodology details, and yaml scripts (with protocol parameters) are available at SAMPL7 GitHub repository (see https://github.com/samplchallenges/ SAMPL7/tree/master/host_guest).

\subsubsection{Participant Calculation Methodologies}

There were a total of 30 submissions from 6 groups for the SAMPL7 host-guest challenge. A good number of methods used alchemical free energy calculations with classical fixed charge (GAFF, GAFF2, CGenFF) and polarizable force fields (AMOEBA), different charging schemes (AM1BCC, RESP), several explicit water models (TIP3P, TIP4P-EW, OPC) and even implicit solvent. Outside of simulation-based free energy methods, quantum mechanical (QM) and QM/MM (molecular mechanics) approaches were also used, and one group employed machine learning. In addition, several groups submitted multiple predictions (particularly for the GDCCs) and the ensuing results are important to provide insight and merit to the methods used here. Participant's submissions with specific details on their methodologies are available in the relevant host-guest system directory in the SAMPL7 GitHub repo (https://github.com/samplchallenges/SAMPL7/tree/master/host_guest/Analysis/Submissions).

\subsection{Experimental Measurements}

The experimental binding data for all host-guest systems are listed in Table 1 and in the SAMPL7 GitHub repo (see https://github. com/samplchallenges/SAMPL7/tree/master/host_guest/Analysis/ExperimentalMeasurements); if there are any updates/changes, the GitHub version is the authoritative one. As mentioned in Section 3.5 a 1:1 binding stoichiometry was confirmed for all host-guest systems. The binding values were determined via ITC and/or NMR typically at 298K. Binding measurements for TrimerTrip were performed in $20 \mathrm{mM}$ sodium phosphate at $\mathrm{pH}$ 7.4. Binding constants for GDCC systems were determined in $10 \mathrm{mM}$ sodium phosphate buffer at pH 11.7. All binding for CD derivative systems were assayed in $25 \mathrm{mM} \mathrm{pH} 6.8$ sodium phosphate buffer. Experimental results suggest all binding was inside the CD-derivative cavity so there is no surface binding. Specific experimental details can be found in the SAMPL7 github repository (see https://github.com/samplchallenges/SAMPL7/tree/master/host guest) and in the relevant experimental papers [58-60] respectively. Binding of one guest (g1) to the GDCC exoOA was undetectable by ITC and NMR ( 1).

\subsection{Statistical/Error Analysis of Challenge}

In general, analysis was performed using Python scripts deposited in the SAMPL7 GitHub repository adapted from previous SAMPL challenges such as the SAMPL6 host-guest challenge, so analysis is extremely similar to what was performed there [87]. All binding free energy prediction sets were compared with experimental data via the following statistical measurements: RMSE (root mean-squared error), $\mathrm{R}^{2}$ (coefficient of determination), $\tau$ (Kendall Tau correlation coefficient), $\mathrm{m}$ (linear regression slope), ME (mean error), and MAE (mean absolute error). Any uncertainty in the error metrics was determined via bootstrapping with replacement, as described previously. Methods for each host-guest system dataset (TrimerTrip, GDCC, and CD derivatives) were only evaluated and compared within the same dataset. In addition, we computed RMSE and ME of methods to each individual host-guest system to ascertain problematic molecules.

The statistical evaluation was separated into two categories, ranked and non-ranked, as described in Section 5.4. All ranked submissions evaluation data, plots, and tables are available at the SAMPL7 GitHub repository (see https://github.com/samplchallenges/ SAMPL7/tree/master/host_guest/Analysis/Accuracy_ranked). Statistical analysis was carried out with and without optional guests. Optional guests were those for which experimental data was already available. In addition, one very poorly performing CD ranked method was not included in much of our analysis because its performance was so poor that it would have made most other methods appear virtually identical, but was included in the non-ranked analysis and in Table 2 (sid 15 or ID AM1BCC/MD/GAFF/TIP4PEW/QMMM). All non-ranked evaluation data, plots, and tables are available in the SAMPL7 GitHub repository (see https://github.com/samplchallenges/SAMPL7/tree/master/host_guest/Analysis/Reference/Accuracy), as is the raw data and the analysis tools.

\section{Results}

Out of the 30 participant submissions, 7 were for TrimerTrip, 16 for the GDCCs, and 9 for the CD derivatives. The TrimerTrip submissions included 3 ranked and 4 non-ranked, GDCCs included 4 ranked and 12 non-ranked, and CD-derivatives had 3 ranked 
Table 1. Experimental binding details for all host-guest systems.

\begin{tabular}{|c|c|c|c|c|c|c|}
\hline ID & name & $K_{a}\left(\mathbf{M}^{-1}\right)$ & $\Delta G(\mathbf{k c a l} / \mathbf{m o l})^{(a)}$ & $\Delta H(\mathrm{kcal} / \mathrm{mol})$ & $T \Delta S(\mathbf{k c a l} / \mathbf{m o l})^{(b)}$ & $n$ \\
\hline clip-g1 & 4-azaniumylbutylammonium & $31000.0 \pm 9000.0$ & $-6.1 \pm 0.2$ & $-6.1 \pm 0.8$ & $0.0 \pm 0.8$ & 0.86 \\
\hline clip-g2 & 5-azaniumylpentylammonium & $1270000.0 \pm 80000.0$ & $-8.32 \pm 0.04$ & $-8.8 \pm 0.3$ & $-0.4 \pm 0.3$ & 1.00 \\
\hline clip-g3 & 6-azaniumylhexylammonium & $24000000.0 \pm 3000000.0$ & $-10.05 \pm 0.07$ & $-10.9 \pm 0.3$ & $-0.8 \pm 0.3$ & 0.90 \\
\hline clip-g15 & trimethyl-[6-(trimethylammonio)hexyl]ammonium & $52000000.0 \pm 4000000.0$ & $-10.52 \pm 0.05$ & $-12.8 \pm 0.4$ & $-2.2 \pm 0.4$ & 0.97 \\
\hline clip-g12 & hexyl(trimethyl)ammonium & $1210000.0 \pm 70000.0$ & $-8.29 \pm 0.03$ & $-8.4 \pm 0.3$ & $-0.1 \pm 0.3$ & 0.94 \\
\hline clip-g5 & 8-azaniumyloctylammonium & $150000000.0 \pm 30000000.0$ & $-11.1 \pm 0.1$ & $-11.4 \pm 0.4$ & $-0.3 \pm 0.4$ & 0.89 \\
\hline clip-g16 & 10-azaniumyldecylammonium & $300000000.0 \pm 100000000.0$ & $-11.5 \pm 0.2$ & $-11.2 \pm 0.4$ & $0.3 \pm 0.4$ & 0.89 \\
\hline clip-g17 & 12-azaniumyldodecylammonium & $500000000.0 \pm 300000000.0$ & $-11.8 \pm 0.4$ & $-10.4 \pm 0.3$ & $1.4 \pm 0.5$ & 0.97 \\
\hline clip-g9 & 1-adamantylammonium & $360000.0 \pm 30000.0$ & $-7.57 \pm 0.05$ & $-4.8 \pm 0.2$ & $2.8 \pm 0.2$ & 0.95 \\
\hline clip-g6 & 1-adamantyl(trimethyl)ammonium & $11000000.0 \pm 2000000.0$ & $-9.6 \pm 0.1$ & $-10.2 \pm 0.4$ & $-0.6 \pm 0.4$ & 0.83 \\
\hline clip-g11 & 1-(1-adamantyl)ethanamine & $4100000.0 \pm 600000.0$ & $-9.02 \pm 0.08$ & $-7.4 \pm 0.3$ & $1.6 \pm 0.3$ & 0.85 \\
\hline clip-g10 & decahydro-2,8,4,6-(epibutane[1,2,3,4]tetrayl)naphthalene-2,6-diaminium & $1000000.0 \pm 100000.0$ & $-8.17 \pm 0.08$ & $-5.8 \pm 0.2$ & $2.3 \pm 0.2$ & 0.99 \\
\hline clip-g8 & [4-(azaniumylmethyl)phenyl]methylammonium & $8500000.0 \pm 700000.0$ & $-9.45 \pm 0.05$ & $-10.6 \pm 0.3$ & $-1.1 \pm 0.3$ & 0.90 \\
\hline clip-g18 & 1-methyl-4-(1-methylpyridin-1-ium-4-yl)pyridin-1-ium & $54000000.0 \pm 8000000.0$ & $-10.55 \pm 0.09$ & $-12.4 \pm 0.4$ & $-1.8 \pm 0.4$ & 0.95 \\
\hline clip-g19 & 4-(1,1-dimethylpiperidin-1-ium-4-yl)-1,1-dimethyl-piperidin-1-ium & $360000000.0 \pm 80000000.0$ & $-11.7 \pm 0.1$ & $-13.6 \pm 0.4$ & $-2.0 \pm 0.5$ & 0.79 \\
\hline clip-g7 & (4-azaniumylcyclohexyl)ammonium & $59000.0 \pm 5000.0$ & $-6.5 \pm 0.05$ & $-6.7 \pm 0.3$ & $-0.2 \pm 0.3$ & 0.83 \\
\hline$\overline{\mathrm{OA}-\mathrm{g} 1}$ & hexanoate & $4400.0 \pm 200.0$ & $-4.97 \pm 0.02$ & $-5.54 \pm 0.1$ & $-0.57 \pm 0.07$ & 1.00 \\
\hline$\overline{\mathrm{OA}-\mathrm{g} 2}$ & 4-chlorobenzoate & $116000.0 \pm 5000.0$ & $-6.91 \pm 0.02$ & $-9.6 \pm 0.3$ & $-2.6 \pm 0.2$ & 1.00 \\
\hline$\overline{\mathrm{OA}-\mathrm{g} 3}$ & (4 S)-4-isopropenylcyclohexene-1-carboxylate & $870000.0 \pm 40000.0$ & $-8.1 \pm 0.02$ & $-12.0 \pm 0.02$ & $-3.9 \pm 0.02$ & 1.00 \\
\hline$\overline{\mathrm{OA}-\mathrm{g} 4}$ & (3 S)-3,7-dimethyloct-6-enoate & $91000.0 \pm 7000.0$ & $-6.76 \pm 0.05$ & $-6.7 \pm 0.2$ & $0.1 \pm 0.1$ & 1.00 \\
\hline$\overline{\mathrm{OA}-\mathrm{g} 5}$ & trimethyl-2-phenylethanaminium & $3000.0 \pm 100.0$ & $-4.73 \pm 0.02$ & $-7.48 \pm 0.05$ & $-2.75 \pm 0.05$ & 1.00 \\
\hline OA-g6 & hexyl(trimethyl)ammonium & $4400.0 \pm 200.0$ & $-4.97 \pm 0.02$ & $-7.3 \pm 0.3$ & $-2.3 \pm 0.3$ & 1.00 \\
\hline OA-g7 & trimethyl-(4-methylcyclohexyl)ammonium & $28000.0 \pm 2000.0$ & $-6.07 \pm 0.05$ & $-5.7 \pm 0.2$ & $0.3 \pm 0.1$ & 1.00 \\
\hline$\overline{\mathrm{OA}-\mathrm{g} 8}$ & 1-adamantyl(trimethyl)ammonium & $1110000.0 \pm 40000.0$ & $-8.25 \pm 0.02$ & $-7.8 \pm 0.2$ & $0.4 \pm 0.1$ & 1.00 \\
\hline exoOA-g1 & hexanoate & $\mathrm{ND} \pm \mathrm{ND}$ & $N D \pm N D$ & $\mathrm{ND} \pm \mathrm{ND}$ & $\mathrm{ND} \pm \mathrm{ND}$ & 1.00 \\
\hline$\overline{\text { exoOA-g2 }}$ & 4-chlorobenzoate & $9.0 \pm 4.0$ & $-1.3 \pm 0.3$ & $\mathrm{ND} \pm \mathrm{ND}$ & $\mathrm{ND} \pm \mathrm{ND}$ & 1.00 \\
\hline$\overline{\text { exoOA-g3 }}$ & (4 S)-4-isopropenylcyclohexene-1-carboxylate & $300.0 \pm 40.0$ & $-3.37 \pm 0.07$ & $-6.0 \pm 0.1$ & $-2.65 \pm 0.07$ & 1.00 \\
\hline exoOA-g4 & (3 S)-3,7-dimethyloct-6-enoate & $440.0 \pm 20.0$ & $-3.61 \pm 0.02$ & $-7.3 \pm 0.7$ & $-3.7 \pm 0.7$ & 1.00 \\
\hline exoOA-g5 & trimethyl-2-phenylethanaminium & $12100.0 \pm 500.0$ & $-5.57 \pm 0.02$ & $-6.17 \pm 0.02$ & $-0.6 \pm 0.02$ & 1.00 \\
\hline exoOA-g6 & hexyl(trimethyl)ammonium & $18900.0 \pm 800.0$ & $-5.83 \pm 0.02$ & $-3.25 \pm 0.02$ & $2.58 \pm 0.02$ & 1.00 \\
\hline exoOA-g7 & trimethyl-(4-methylcyclohexyl)ammonium & $130000.0 \pm 20000.0$ & $-6.98 \pm 0.1$ & $-4.97 \pm 0.07$ & $2.01 \pm 0.05$ & 1.00 \\
\hline exoOA-g8 & 1-adamantyl(trimethyl)ammonium & $420000.0 \pm 20000.0$ & $-7.67 \pm 0.02$ & $-5.04 \pm 0.05$ & $2.63 \pm 0.02$ & 1.00 \\
\hline$\overline{\mathrm{bCD}-\mathrm{g} 1}$ & trans-4-methylcyclohexanol & $2100.0 \pm 100.0$ & $-4.52 \pm 0.03$ & $-2.6 \pm 0.2$ & $2.0 \pm 0.2$ & 0.88 \\
\hline bCD-g2 & R-rimantadine & $35000.0 \pm 3000.0$ & $-6.2 \pm 0.04$ & $-10.4 \pm 0.7$ & $-4.2 \pm 0.7$ & 1.00 \\
\hline MGLab_8-g1 & trans-4-methylcyclohexanol & $260.0 \pm 20.0$ & $-3.3 \pm 0.05$ & $-1.8 \pm 0.4$ & $1.5 \pm 0.4$ & 0.89 \\
\hline MGLab_8-g2 & R-rimantadine & $830.0 \pm 50.0$ & $-3.98 \pm 0.04$ & $-6.9 \pm 0.5$ & $-2.9 \pm 0.5$ & 1.03 \\
\hline MGLab_9-g1 & trans-4-methylcyclohexanol & $210.0 \pm 20.0$ & $-3.17 \pm 0.05$ & $-2.7 \pm 0.8$ & $0.4 \pm 0.8$ & 0.88 \\
\hline MGLab_9-g2 & R-rimantadine & $700.0 \pm 40.0$ & $-3.88 \pm 0.03$ & $-9.0 \pm 0.6$ & $-5.2 \pm 0.6$ & 1.00 \\
\hline MGLab_19-g1 & trans-4-methylcyclohexanol & $210.0 \pm 20.0$ & $-3.18 \pm 0.04$ & $-2.1 \pm 0.2$ & $1.1 \pm 0.2$ & 0.83 \\
\hline MGLab_19-g2 & R-rimantadine & $320.0 \pm 20.0$ & $-3.41 \pm 0.04$ & $-11.0 \pm 1.0$ & $-8.0 \pm 1.0$ & 0.94 \\
\hline MGLab_23-g1 & trans-4-methylcyclohexanol & $220.0 \pm 20.0$ & $-3.2 \pm 0.05$ & $-3.0 \pm 1.0$ & $0.0 \pm 1.0$ & 0.76 \\
\hline MGLab_23-g2 & R-rimantadine & $1510.0 \pm 90.0$ & $-4.33 \pm 0.04$ & $-7.6 \pm 0.5$ & $-3.3 \pm 0.5$ & 0.96 \\
\hline MGLab_24-g1 & trans-4-methylcyclohexanol & $280.0 \pm 20.0$ & $-3.34 \pm 0.05$ & $-1.6 \pm 0.2$ & $1.7 \pm 0.2$ & 0.92 \\
\hline MGLab_24-g2 & R-rimantadine & $1100.0 \pm 70.0$ & $-4.15 \pm 0.04$ & $-8.6 \pm 0.6$ & $-4.5 \pm 0.6$ & 1.03 \\
\hline MGLab_34-g1 & trans-4-methylcyclohexanol & $700.0 \pm 100.0$ & $-3.85 \pm 0.09$ & $-3.7 \pm 0.3$ & $0.1 \pm 0.3$ & 0.81 \\
\hline MGLab_34-g2 & R-rimantadine & $11000.0 \pm 7000.0$ & $-5.5 \pm 0.4$ & $-9.0 \pm 2.0$ & $-3.0 \pm 2.0$ & 0.99 \\
\hline MGLab_35-g1 & trans-4-methylcyclohexanol & $2300.0 \pm 200.0$ & $-4.58 \pm 0.05$ & $-4.5 \pm 0.3$ & $0.1 \pm 0.3$ & 0.85 \\
\hline MGLab_35-g2 & R-rimantadine & $27000.0 \pm 2000.0$ & $-6.04 \pm 0.04$ & $-7.3 \pm 0.5$ & $-1.2 \pm 0.5$ & 0.78 \\
\hline MGLab_36-g1 & trans-4-methylcyclohexanol & $200.0 \pm 10.0$ & $-3.15 \pm 0.04$ & $-3.0 \pm 0.3$ & $0.1 \pm 0.3$ & 0.87 \\
\hline MGLab_36-g2 & R-rimantadine & $350.0 \pm 20.0$ & $-3.48 \pm 0.04$ & $-11.0 \pm 1.0$ & $-7.0 \pm 1.0$ & 0.84 \\
\hline
\end{tabular}

All quantities are reported as point estimate \pm statistical error from the ITC data fitting procedure. The upper bound (1\%) was used for errors reported to be $<1 \%$. We also included a $3 \%$ relative uncertainty in the titrant concentration assuming the stoichiometry coefficient to be fitted to the ITC data [1] for the Isaacs (TrimerTrip) and Gilson (cyclodextrin derivatives) datasets, where concentration error had not been factored in to the original error estimates. For the OA/exo-OA sets, provided uncertainties already included concentration error. In some cases, exoOA-g1 binding constants were not detected (ND) by ITC or H NMR. Binding of guest g2 to exoOA was very weak so only $\mathrm{H}$ NMR spectroscopy could produce reliable free energy data.

$\left(^{(a}\right)$ Statistical errors were propagated from the $K_{a}$ measurements.

$\left(^{b}\right)$ All experiments were performed at $298 \mathrm{~K}$.

$\left(^{c}\right)$ Units of $\mathrm{M}^{-2}$.

${ }^{(d)}$ Units of $\mathrm{M}^{-3}$.

and 6 non-ranked (Table 5). For a large portion of methods submitted, docking was used to obtain starting structures. General classical fixed charge force fields with no explicit polarization treatment were commonly used, as has become common in SAMPL host-guest challenges. Most simulation-based methods used explicit solvent, and used the TIP3P, TIP4PEW, and OPC water models. For this challenge one method did explicitly treat electronic polarizability. In addition, quantum mechanical methods were used in 2 of the datasets (GDCC and Cyclodextrin). Alchemical free energy techniques were employed in many cases, with anal- 
ysis done via thermodynamic integration (TI) and Bennett acceptance ratio (BAR) for equilibrium calculations. Nonequilibrium approaches were also employed, such as using the fast switching double annihilation method (FSDAM) approach [88]. Other simulation-based methods used the MM/PBSA (Molecular mechanics Poisson Boltzmann/solvent accessible surface area) or MM/GBSA (Molecular mechanics Generalized Born/solvent accessible surface area) endpoint methods, and another submission used self association molecular dynamics (SA-MD). Moreover, for the GDDC dataset some methods used a machine learning approach or made empirical corrections based on literature data. Here we introduce the results for all ranked methods separated by host-guest system dataset, give statistics for binding of individual host-guest systems averaged across methods, and lastly examine analysis of non-ranked methods including our own reference calculations.

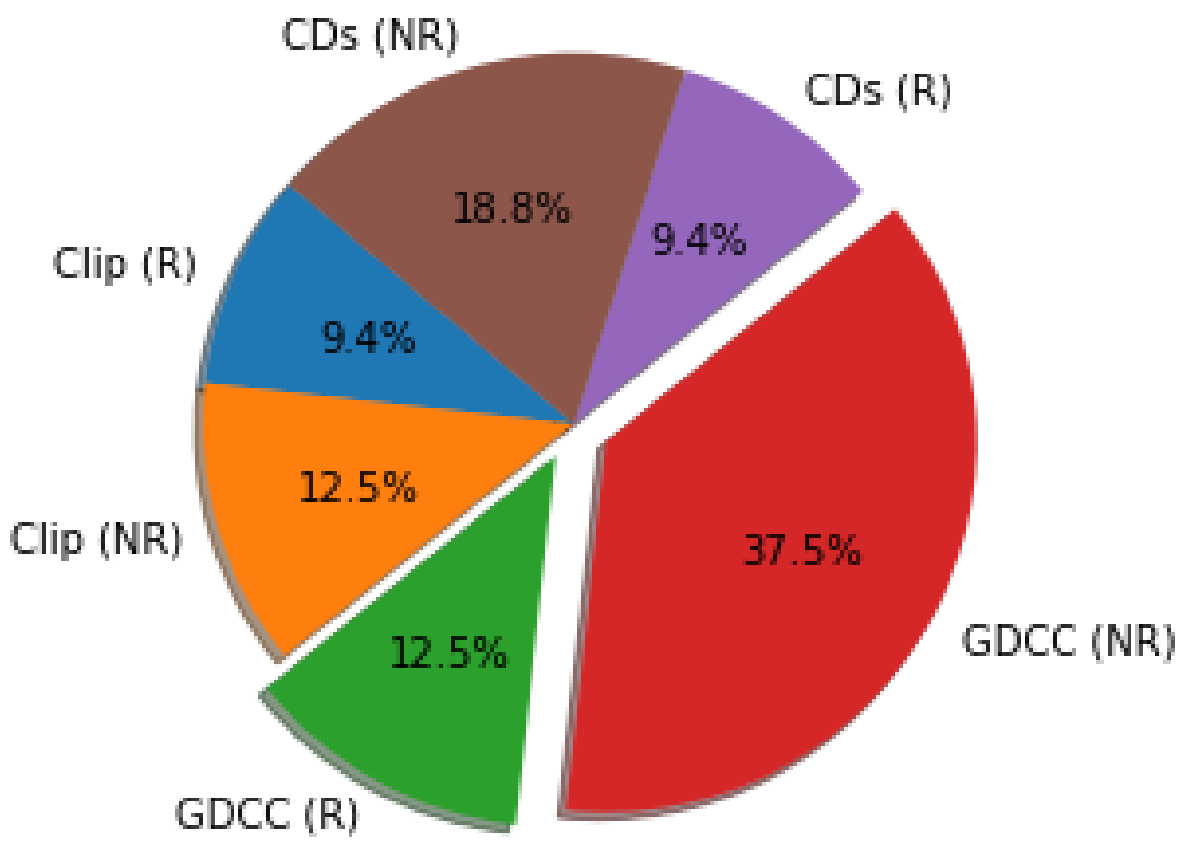

Figure 5. SAMPL7 submission breakdown. The SAMPL7 challenge saw 7 TrimerTrip submissions, of which 3 were ranked (blue) and 4 were non-ranked (orange). There were 16 GDCC submissions, with 4 ranked (green) and 12 nonranked (red), and 7 CD submissions, with 3 ranked (purple) and 4 nonranked (brown).

\subsection{Ranked Submissions}

\subsubsection{TrimerTrip}

Statistical analysis of the 3 sets of ranked absolute binding free energy predictions for the TrimerTrip dataset are summarized in Table 2 and Figure 6. All methods used explicit solvent. Fixed-charge methods used GAFF and GAFF2, along with the TIP3P or OPC water models (the method called MD/DOCKING/GAFF/xtb-GNF/ used GAFF with TIP3P, while FSDAM/GAFF2/OPC3 used GAFF2 with OPC). The third method in this category, AMOEBA/DDM/BAR, used the AMOEBA force field, which explicitly treats polarizability and was consistently the top performing method with values of $2.76 \mathrm{kcal} / \mathrm{mol}, 0.50,1.25$, and $0.47 \mathrm{in}$ terms of RMSE, $\mathrm{R}^{2}$, slope (m), and $\tau$ respectively (Figure 6). The mean error (ME) for this AMOEBA submission was modestly larger in magnitude than one of the other ranked submissions, but in all other respects its performance was superior. Full statistics are in Table 3. AMOEBA-based approaches also perform well in the GDCC category, as we will see below.

Here, the AMOEBA/DDM/BAR method predicted 10/16 binding affinities within $2 \mathrm{kcal} / \mathrm{mol}$, the majority of these being within $1 \mathrm{kcal} / \mathrm{mol}$ (as discussed in the SAMPL7 virtual workshop [43]; full data available in our GitHub repository). The outliers for this method were clip-g6, clip-g7, clip-g8, clip-g9, clip-g11, and clip-g17, of which binding affinities were predicted to be too unfavorable. The FSDAM/GAFF2/OPC3 method predicted 10/16 within $2 \mathrm{kcal} / \mathrm{mol}$ and host-guest system outliers were clip-g3, clipg8, clip-g10, clip-g11, clip-g16, and clip-g18. The other ranked submission, MD/DOCKING/GAFF/xtb-GNF, predicted 5/16 binding affinities within $2 \mathrm{kcal} / \mathrm{mol}$, with 4 of those 5 being within $1 \mathrm{kcal} / \mathrm{mol}$. Interestingly, all of the predictions within $2 \mathrm{kcal} / \mathrm{mol}$ used starting poses generated not by docking, but by using SA-MD. The SA-MD approach makes the assumption that the host is not 
in its proper conformation and the host and guest are allowed to associate on their own [43]. It would be interesting to see the predictive accuracy of this approach on the remaining TrimerTrip host-guest systems, and which systems if any prove to be troublesome.
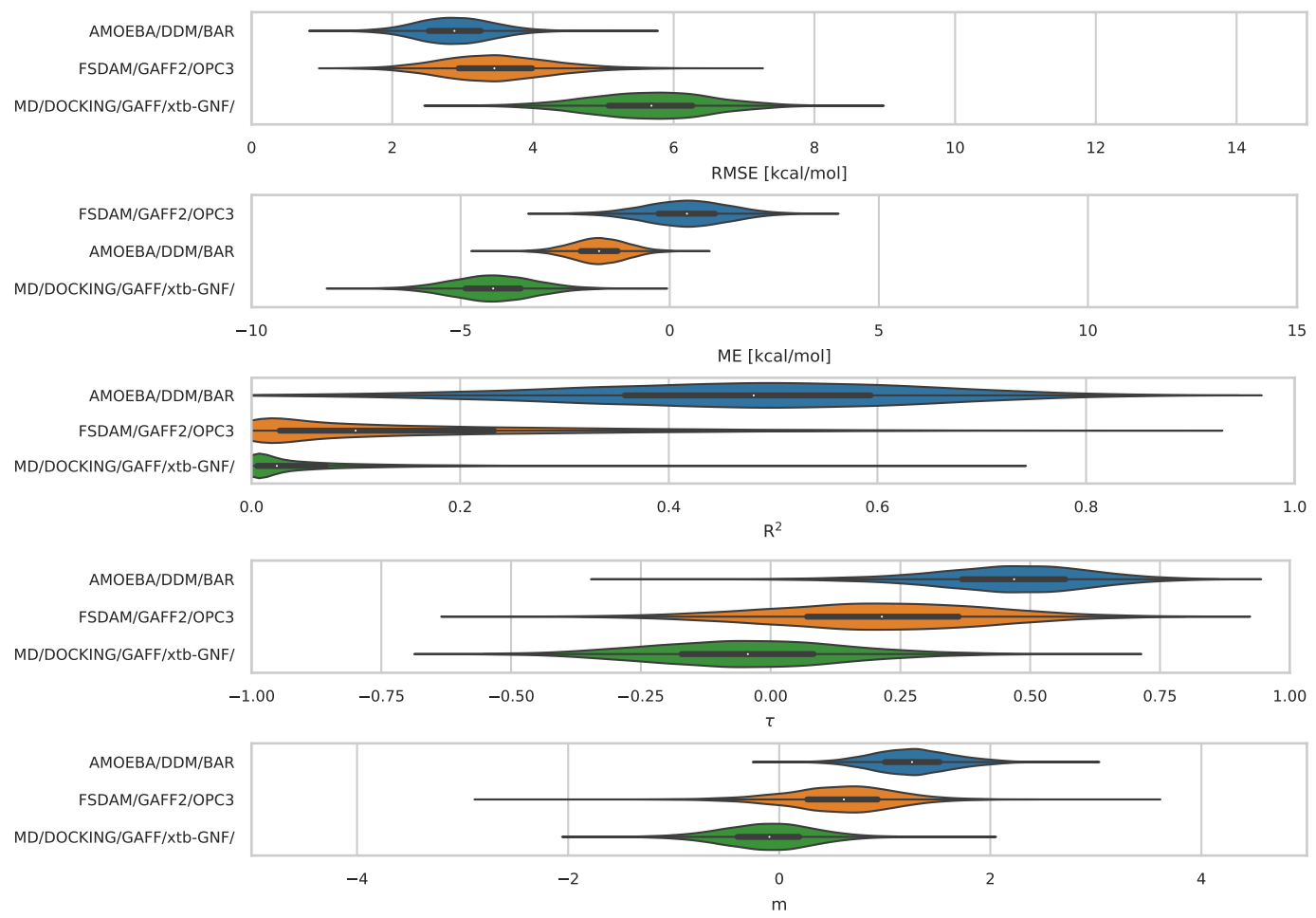

Figure 6. TrimerTrip Error Metrics for Ranked Methods TrimerTrip submissions ordered based on the median indicated by the white circle in the violin plots. The violin plots were generated by bootstrapping samples with replacement, and the plots describe the shape of the sampling distribution for each prediction. The black horizontal bar represents the first and third quartiles. From top to bottom the error metrics are RMSE, $\mathrm{ME}, \mathrm{R}^{2}, \tau$, and slope $(\mathrm{m})$.

Two of these methods, AMOEBA/DDM/BAR and MD/DOCKING/GAFF/xtb-GNF, tended to yield binding free energies which were too unfavorable while the FSDAM/GAFF2/OPC3 ranked method was too favorable (Figure 6). Thus, most predictions with errors larger in $2 \mathrm{kcal} / \mathrm{mol}$ in magnitude err in the direction of not predicting binding to be favorable enough, especially with AMOEBA/DDM/BAR and MD/DOCKING/GAFF/xtb-GNF are underpredicted (Figure 7). On the other hand, as shown by Figures 6 and 7, the FSDAM/GAFF2/OPC3 method errs in both directions more frequently.
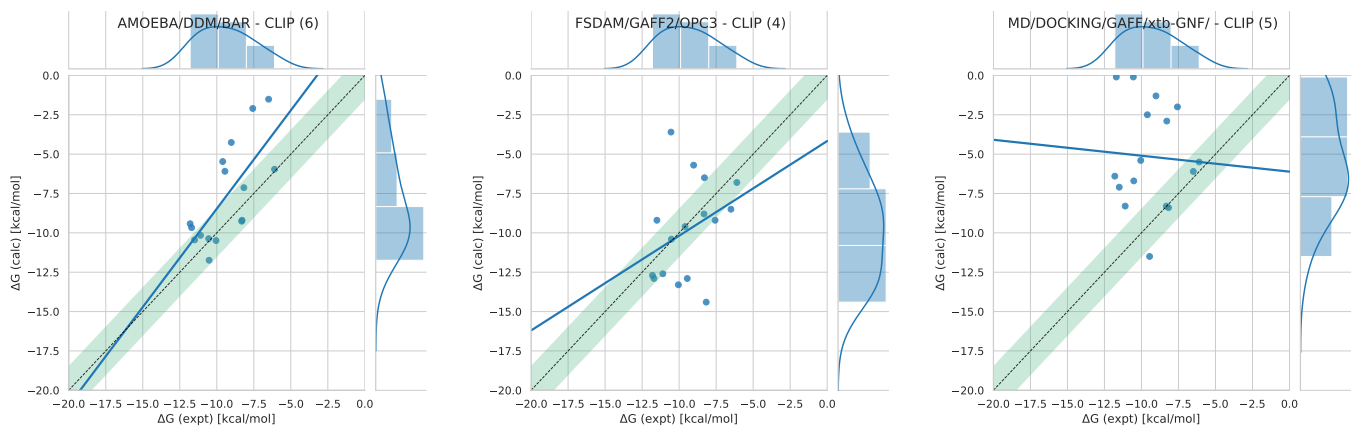

Figure 7. Correlation plots for TrimerTrip ranked submissions Correlation plots comparing (Left to Right) $A M O E B A / D D M / B A R$, $F S$ $D A M / G A F F 2 / O P C 3$, and MD/DOCKING/GAFF/xtb-GNF ranked predictions with experimental values for the TrimerTrip dataset. The $\mathrm{R}^{2}$ and slope for each ranked prediction were 0.50 and $1.25,0.12$ and 0.60 , and 0.00 and -0.10 respectively.

We sought to determine whether some hosts/guests are particularly challenging to predict, across all ranked methods, so we examined the RMSE and ME by host and guest for ranked free energy predictions for all individual host-guest systems. This is 
shown in Figure 8. The ranked predictions of all methods for the TrimerTrip/"clip" host-guest systems (shown in blue in Figure 8) were in general the most problematic, especially clip-g6, clip-g9, clip-g10, clip-g11, clip-g18, and clip-g19 which had an RMSE of about $4 \mathrm{kcal} / \mathrm{mol}$ or greater. All of the guests with an adamantane moiety fall within this list of "problematic" molecules. The computed binding affinities for these host-guest systems are mostly too weak with $\Delta \mathrm{G} \mathrm{ME} \mathrm{of}-2.5 \mathrm{kcal} / \mathrm{mol}$ or greater, the exception being clip-g10 which was predicted to be too favorable with a $\Delta \mathrm{G} \mathrm{ME}$ of $2 \mathrm{kcal} / \mathrm{mol}$.
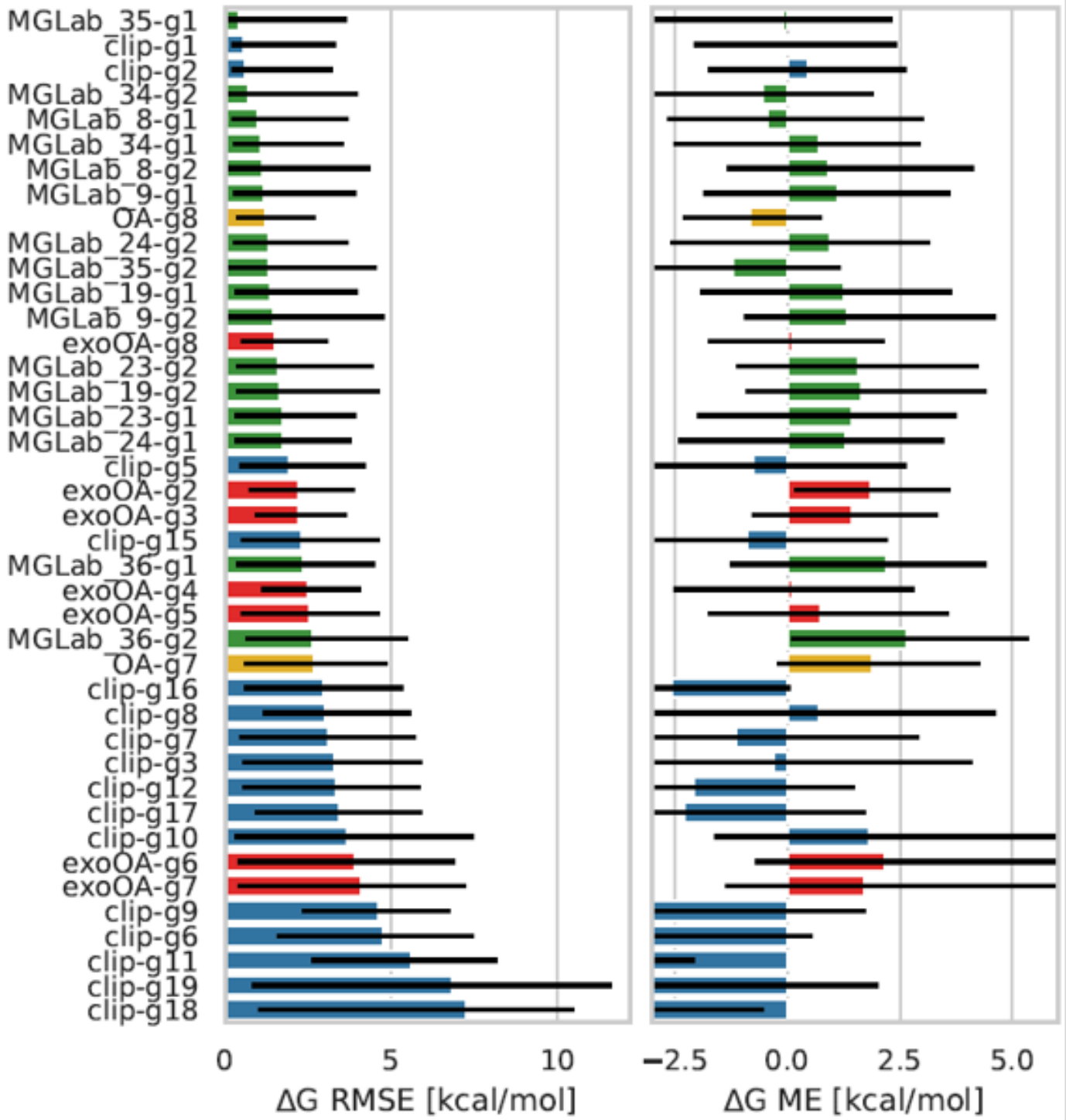

Figure 8. RMSE and ME statistics by host-guest system for ranked methods. Shown are free energy error statistics by host-guest system, across methods/participants. The $\Delta G$ root mean square error (RMSE) and mean signed error (ME) were computed via bootstrapping with replacement for all host-guest systems (except optional systems OA-g1, OA-g2, OA-g3, OA-g4, OA-g5, OA-g6, bCD-g1, and bCD-g2) and includes all ranked methods submitted (except AM1-BCC/MD/GAFF/TIP4PEW/QMMM method for the cyclodextrin dataset). The black error bars represent the 95-percentile bootstrap confidence intervals. The host-guest datasets for the SAMPL7 challenge were TrimerTrip (blue), OA (yellow), exo-OA (red), and cyclodextrin derivatives (green)

Overall for the TrimerTrip/clip-based systems, when we consider both ranked and non-ranked submissions, we believe the results suggest that any combination of the following may be limiting predictive accuracy: (a) chosen host conformer, (b) guest binding mode, (c) chosen energy model, and (d) water model. More specifically the general performance of the AMOEBA force field pointed towards polarization and/or shielding effects being important, especially as the guest becomes more hydrophobic, but the AMOEBA work also suggested host sampling could be an important issue. 
Table 2. Error metrics for ranked method submission of absolute binding free energy calculations of all hostguest systems. The root mean square error (RMSE), mean absolute error (MAE), signed mean error (ME), coefficient of correlation $\left(R^{2}\right)$, slope $(m)$, and Kendall's rank correlation coefficient $(\tau)$ were computed, with confidence intervals from bootstrapping with replacement. All four datasets (TrimerTrip, GDCC-exoOA, Cyclodextrin derivatives), and the fourth dataset which includes both OA and exo-OA ranked submissions (GDCC-OA and exoOA) are included. Statistical values in this table do not include optional host-guest systems OA-g1, OA-g2, OA-g3, OA-g4, OA-g5, OA-g6, bCD-g1, and bCD-g2, for which values had been released previously.

\begin{tabular}{|c|c|c|c|c|c|c|c|}
\hline ID & sid & RMSE [kcal/mol] & MAE [kcal/mol] & ME [kcal/mol] & $\mathbf{R}^{2}$ & m & $\tau$ \\
\hline \multicolumn{8}{|l|}{ TrimerTrip } \\
\hline AMOEBA/DDM/BAR & 6 & $2.76[1.83,3.98]$ & $2.12[1.35,3.33]$ & $-1.69[-2.98,-0.44]$ & $0.50[0.13,0.77]$ & $1.25[0.53,2.06]$ & $0.47[0.12,0.74]$ \\
\hline FSDAM/GAFF2/OPC3 & 4 & $2.97[2.11,5.13]$ & $2.24[1.62,4.22]$ & $0.43[-1.59,2.33]$ & $0.12[0.00,0.56]$ & $0.60[-0.51,1.60]$ & $0.24[-0.23,0.61]$ \\
\hline MD/DOCKING/GAFF/Xtb-GNF/ & 5 & $5.65[3.87,7.36]$ & $4.51[3.01,6.40]$ & $-4.23[-6.19,-2.23]$ & $0.00[0.00,0.26]$ & $-0.10[-1.02,0.80]$ & $-0.05[-0.41,0.35]$ \\
\hline \multicolumn{8}{|l|}{$\overline{G D C C}-O A$ and exOOA } \\
\hline RESP/GAFF/MMPBSA-Cor & 20 & $1.24[0.76,2.46]$ & $0.95[0.59,2.15]$ & $0.94[-0.13,1.99]$ & $0.94[0.11,0.97]$ & $0.65[0.18,1.14]$ & $0.83[0.03,1.00]$ \\
\hline AMOEBA/DDM/BAR & 29 & $1.25[0.68,2.53]$ & $0.92[0.54,2.12]$ & $-0.36[-1.54,0.83]$ & $0.80[0.34,0.97]$ & $1.11[0.57,1.97]$ & $0.72[0.18,1.00]$ \\
\hline xtb-GNF/Machine Learning/CORINA_MD & 28 & $2.26[1.39,3.44]$ & $1.91[1.10,3.12]$ & $0.37[-1.31,2.06]$ & $0.01[0.00,0.78]$ & $0.04[-0.58,0.54]$ & $0.06[-0.64,0.81]$ \\
\hline B2PLYPD3/SMD_QZ-R & 23 & $4.52[2.52,6.39]$ & $3.70[1.96,5.67]$ & $3.15[0.84,5.44]$ & $0.49[0.03,0.93]$ & $1.43[-0.11,2.98]$ & $0.37[-0.31,0.87]$ \\
\hline \multicolumn{8}{|l|}{$\overline{\text { GDCC - exoOA }}$} \\
\hline AMOEBA/DDM/BAR & 29 & $1.27[0.56,2.72]$ & $0.91[0.45,2.31]$ & $-0.66[-1.98,0.61]$ & $0.81[0.30,0.99]$ & $1.05[0.45,2.12]$ & $0.71[0.05,1.00]$ \\
\hline RESP/GAFF/MMPBSA-Cor & 20 & $1.32[0.68,2.65]$ & $1.03[0.54,2.34]$ & $1.01[-0.18,2.20]$ & $0.95[0.04,0.99]$ & $0.61[0.04,1.20]$ & $0.81[-0.14,1.00]$ \\
\hline xtb-GNF/Machine Learning/CORINA MD & 28 & $2.43[1.40,3.71]$ & $2.11[1.10,3.42]$ & $0.82[-1.12,2.77]$ & $0.00[0.00,0.91]$ & $0.01[-0.81,0.57]$ & $0.05[-0.78,1.00]$ \\
\hline B2PLYPD3/SMD_QZ-R & 23 & $4.76[2.26,6.93]$ & $3.90[1.81,6.26]$ & $3.50[0.91,6.12]$ & $0.72[0.24,0.99]$ & $1.97[0.88,3.77]$ & $0.59[-0.06,1.00]$ \\
\hline \multicolumn{8}{|l|}{ Cyclodextrin derivatives } \\
\hline FSDAM/GAFF2/OPC3_ranked & 12 & $1.28[1.32,3.51]$ & $1.04[1.04,2.95]$ & $0.63[-0.84,2.10]$ & $0.01[0.00,0.50]$ & $0.12[-1.62,2.30]$ & $0.21[-0.46,0.57]$ \\
\hline Noneq/Alchemy/consensus & 27 & $1.70[1.27,2.28]$ & $1.48[1.03,2.04]$ & $1.21[0.52,1.87]$ & $0.02[0.00,0.29]$ & $0.16[-0.48,0.93]$ & $-0.02[-0.43,0.45]$ \\
\hline AM1-BCC/MD/GAFF/TIP4PEW/QMMM & 15 & $46.62[22.85,65.69]$ & $32.00[17.92,49.22]$ & $31.27[16.89,48.87]$ & $0.04[0.00,0.33]$ & $7.62[-3.31,30.72]$ & $0.24[-0.13,0.52]$ \\
\hline
\end{tabular}

\subsubsection{GDCC}

The GDCC dataset, which includes OA and exo-OA host-guest systems, had the most submissions, probably because this host is familiar to many participants since it has formed part of a variety of previous SAMPL challenges. The statistical analysis of 4 sets of ranked methods are shown in Figure 9. For the entire GDCC dataset there was not a clear top performing method in terms of RMSE, $\mathrm{R}^{2}, \tau$, and slope, but RESP/GAFF/MMPBSA-Cor and AMOEBA/DDM/BAR methods were the two top performing methods. Again, the $A M O E B A / D D M / B A R$ method emerged among the top performers, but unlike in the TrimerTrip challenge it is not the top method by all error metrics. The RESP/GAFF/MMPBSA-Cor method had the top $\triangle \mathrm{G} \mathrm{RMSE,} \mathrm{R}^{2}$, and $\tau$ values of $1.24 \mathrm{kcal} / \mathrm{mol}, 0.94$, and 0.83 respectively. Essentially, the latter approach seems to have done slightly better at ranking compounds for binding than the AMOEBA-based approach, but with a slope which is systematically incorrect. Full performance statistics are in Table 2.

Figure 10 shows performance of ranked methods relative to experiment. In general, the AMOEBA/DDM/BAR method tends to yield GDCC binding free energies which are too unfavorable, while all other ranked methods tend to predict binding free energies that are too favorable. The AMOEBA/DDM/BAR method gave calculated values that most directly tracked with experimental ones, as evidenced by a slope, $m$, of 1.11 . With this approach, only exoOA-g4 had an error larger than $2 \mathrm{kcal} / \mathrm{mol}$. The exoOA-g2 host-guest system was the only outlier for the RESP/GAFF/MMPBSA-Cor method, and the participants suggested this was likely due to guest g2 containing a chlorine atom. The QM-based method B2PLYPD3/SMD QZ-R had large prediction errors in more cases than any other method, in part because it overestimated the dynamic range of predictions and led to calculated binding free energies that were often far too negative. The xtb-GNF/MachineLearning/CORINA MD had smaller errors, but the correlation between calculated and experimental free energies was poor.

The xtb-GNF/MachineLearning/CORINA MD, RESP/GAFF/MMPBSA-Cor, and AMOEBA/DDM/BAR methods have greater prediction errors for systems with negatively charged guests, which could potentially relate to the challenges alchemical methods have with charged guests (Section 3.1). Both xtb-GNF/MachineLearning/CORINA MD and RESP/GAFF/MMPBSA-Cor use the GAFF energy model, and its combination with explicit fixed charge water models typically results in predicted free energies that are too favorable (particularly, prior work has shown that GAFF with TIP3P leads to a consistent error in this direction for guests containing carboxylates and alcohols [49]). This is exactly the case here for systems with guests containing a carboxylate for the $x$ tb-GNF/MachineLearning/CORINA MD method, where an AM1-BCC charging scheme, explicit TIP3P water, and GAFF energy model is used. The RESP/GAFF/MMPBSA-Cor method also used the GAFF energy model, but with implicit (PB/SA) water and a RESP charging scheme. During the SAMPL7 virtual workshop [43] the RESP/GAFF/MMPBSA-Cor participants noted that in their methodology comparison of RESP and AM1-BCC charging schemes, RESP resulted in better accuracy; it would be interesting to know if this holds true more generally. For the the $A M O E B A / D D M / B A R$ method, the single outlier was exoOA-g4, with a $\triangle \mathrm{G}$ prediction error of $2.5 \mathrm{kcal} / \mathrm{mol}$. 

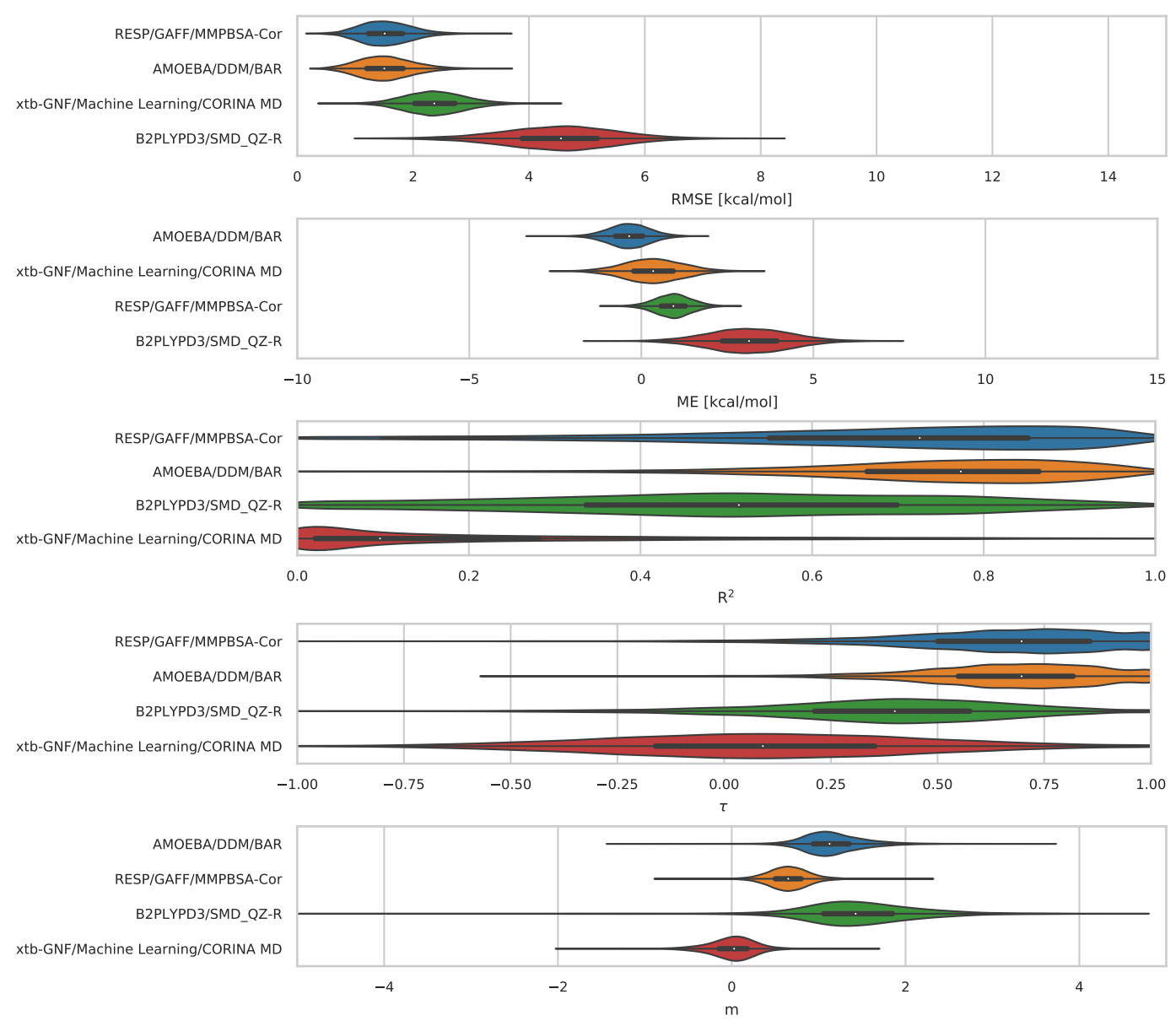

Figure 9. GDCC Error Metrics for Ranked Methods. Shown is accuracy of GDCC submissions, with the median value for each metric indicated by the white circle in the violin plots. The violin plots were generated by bootstrapping samples with replacement, and the plots describe the shape of the sampling distribution for each prediction. The black horizontal bar represents the first and third quartiles. From top to bottom the error metrics are RMSE, ME, $\mathrm{R}^{2}, \tau$, and slope $(\mathrm{m})$.

The Ponder group's data suggests that the quality of torsional parameters for the upper rim's diphenyl ether torsions can change predictions by $3-4 \mathrm{kcal} / \mathrm{mol}$. In our reference calculations, we observe this guest folding in on itself and becoming effectively bulkier, which may mean host torsional parameters play a larger role for this particular guest.

On the other hand, the B2PLYPD3/SMD-QZ-R quantum method had larger prediction errors for guests with a positive charge. Particularly, the methods $\Delta G$ prediction error for exoOA-g6 and exoOA-g7 was $10 \mathrm{kcal} / \mathrm{mol}$, and $5 \mathrm{kcal} / \mathrm{mol}$ for exoOA-g5. Similarly, for the OA-g7 system which contains a positive guest, the method had a $\Delta G$ prediction error of $5 \mathrm{kcal} / \mathrm{mol}$. These prediction errors substantially affected molecule statistics (Figure 8 ) for these systems.

\subsubsection{Cyclodextrins}

Method performance on the CD dataset is shown in Figure 12. Partly because of the narrow range of experimental binding free energies, we observe little difference in performance between the two better performing ranked methods. The third ranked submission AM1-BCC/MD/GAFF/TIP4PEW/QMMM method was not included in these plots because the range of binding free energies is so dramatically overestimated (Figure 13) that including it in the graph makes performance of the other two methods appear identical. In this analysis, optional systems bCD-g1 and bCD-g2 are not included, since these free energies have been previously reported. Of the two better performing techniques here (FSDAM/GAFF2/OPC3 and Noneq/Alchemy/consensus), performance was remarkably similar, as were the nonequilibrium free energy techniques employed. The third method - which typically predicted binding to be far too strong - was the AM1-BC/MD/GAFF/TIP4PEW/QMMM method, which had a ME and slope of $31.27 \mathrm{kcal} / \mathrm{mol}$ and 7.62 respectively. Since the GAFF force field is shared between this method and one of the more successful methods, it seems likely the larger error in this case is due to the QM/MM energy calculation approach. 

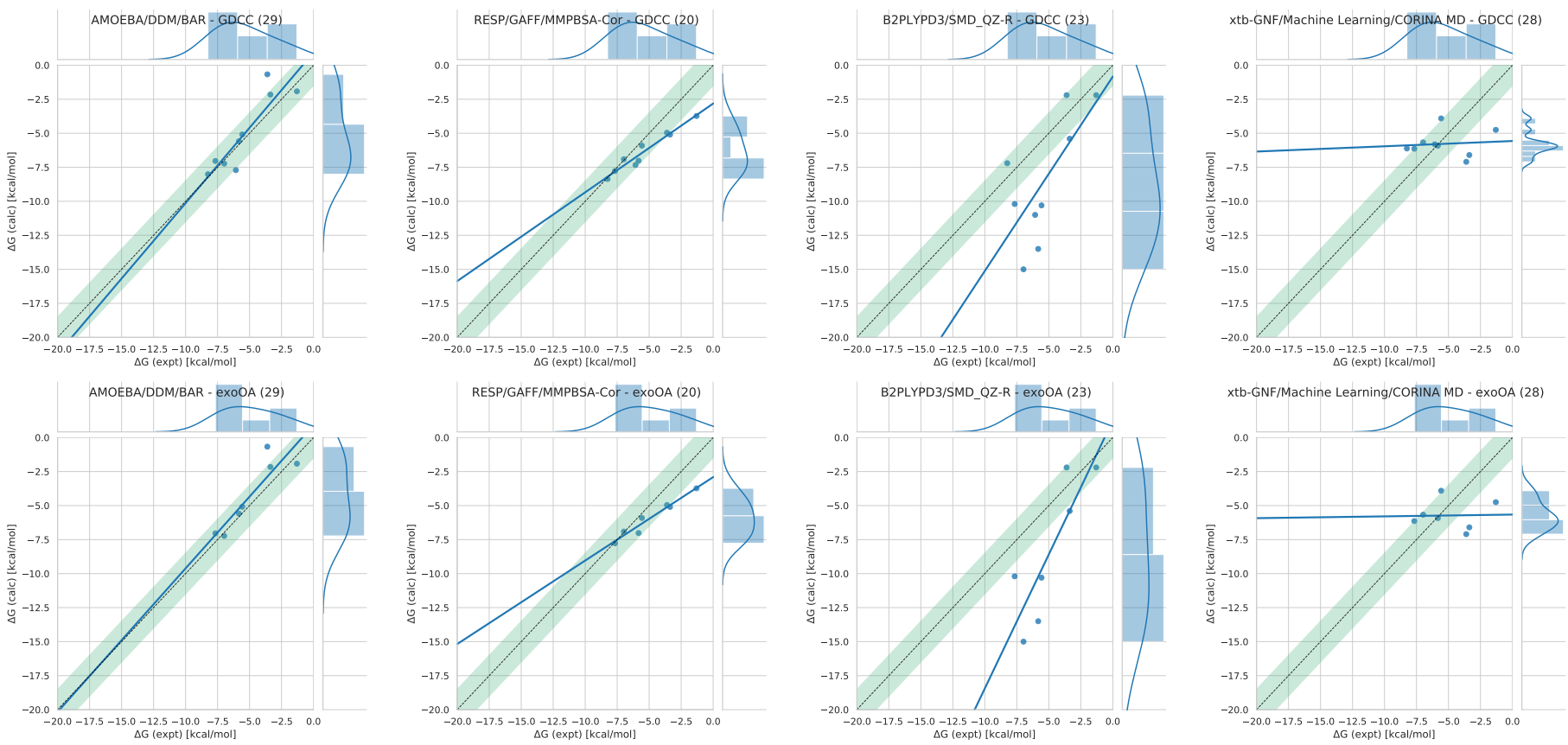

Figure 10. Correlation plots for GDCC, OA, and exo-OA ranked submissions. Correlation plots comparing (Left to Right) $A M O E B A / D D M / B A R$, RESP/GAFF/MMPBSA-Cor, B2PLYPD3/SMD_QZ-R, and xtb-GNF/Machine Learning/CORINA MD ranked predictions with experimental values for the GDCC (top row) and exo-OA (bottom row). The AMOEBA/DDM/BAR approach performed particularly well by a variety of metrics, as did RESP/GAFF/MMPBSA-Cor. The former had the slope closest to 1 and its RMS error was among the lowest, whereas the latter performed better on error and correlation metrics but had a lower slope which was systematically off. (See Table 3)

\subsection{Non-Ranked Submissions}

\subsubsection{TrimerTrip}

Several groups submitted multiple methods, often changing just one aspect of their approach. Such tests can help establish which aspects of an approach impact accuracy and how. Results for all submissions, ranked and non-ranked, are shown in Table 3. Results are listed in ascending order based on $\Delta G$ RMSE values. Here we discuss the analysis of these results and what we find that we can learn from them.

On TrimerTrip, two non-ranked submissions with the AMOEBA force field (AMOEBA/DDM/BAR/ALT1 and AMOEBA/DDM/BAR/ALT2) were used to examine how the selected TrimerTrip conformer impacts calculated binding free energies. The submitters examined so-called "indented" and "overlapping" host conformers which they identified in exploratory simulations. They find that these do not interconvert on the timescale of typical free energy calculations. The indented conformer resembles the annealed structure we provided in the SAMPL7 GitHub repository, while the overlapping conformer is very similar to the previously published structure of the unligated clip analog with four glycoluril units [43] and interconverts relatively rapidly with a so-called "spiral" conformer with staggered triptycene walls [43].

Since some of these conformations interconvert slowly, this introduces a conformation-dependence in calculated binding free energies. Not only may guests bind differently to the different host conformations, but calculated binding free energies depend on the host conformation because different unligated host conformations have different free energies in solution (e.g. some will likely be more strained/less populated than others) and do not relax back on simulation timescales.

To address these issues, the Ponder group used a separate set of free energy calculations to compute the binding free energy to each host conformation (indented and overlapping). However, the resulting free energies are sensitive to the choice of host conformation, since it does not relax back on simulation timescales, so they needed to estimate the relative free energy of the two unligated host conformations. In their submissions, their ranked AMOEBA/DDM/BAR submission assumes the indented TrimerTrip conformer is $2.84 \mathrm{kcal} / \mathrm{mol}$ more stable than the overlapping conformer, while the AMOEBA/DDM/BAR/ALT1 method assumes the overlapping conformer is $2.41 \mathrm{kcal} / \mathrm{mol}$ more stable than the indented, and the AMOEBA/DDM/BAR/ALT2 assumes both conformers are equal in free energy. The non-ranked AMOEBA submissions performed better than their ranked counterpart by almost all of the error metrics 3. Most of the improvement was attributed to better agreement for clip-g6, clip-g7, clip-g8, 

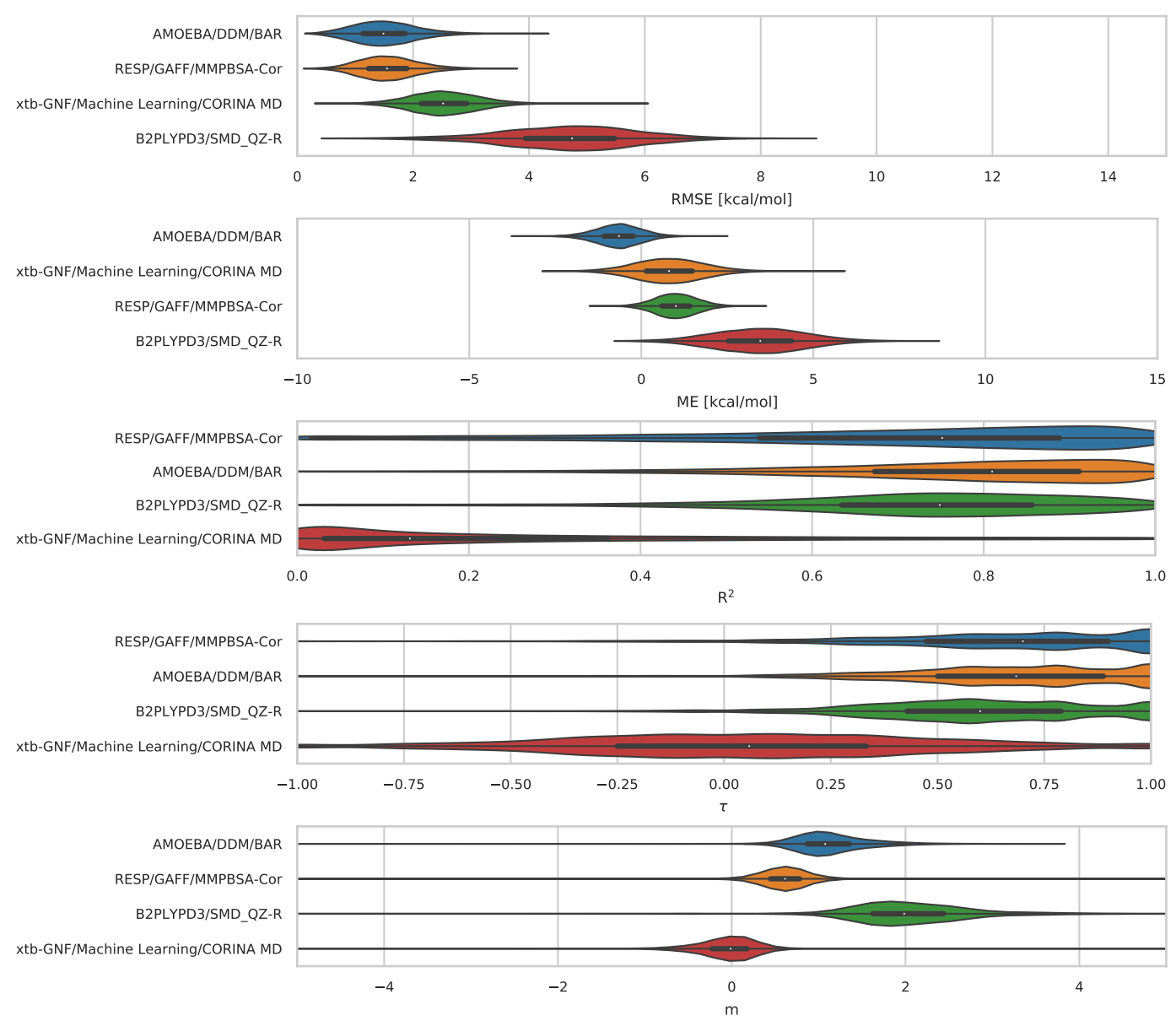

Figure 11. exo-OA Error Metrics for Ranked Methods. Shown are exo-OA methods, with the median indicated by the white circle in the violin plots. The violin plots for RMSE, ME, $\mathrm{R}^{2}$, $\tau$, and slope describe the shape of the sampling distribution after bootstrapping for each method. The black horizontal bar represents the first and third quartiles. From top to bottom the error metrics are RMSE, ME, $\mathrm{R}^{2}, \tau$, and slope (m).

clip-g9, and clip-g11 when using an overlapping conformer. The Ponder group suggests that these results indicate that larger and bulkier guests prefer the overlap/spiral conformer(s), while the smaller guests prefer the indented conformer. TrimerTrip's flexibility seems to allow it to alter its conformation when binding guests of various size - a feature we noticed in our reference calculations and one also reported by the Ponder group [43].

Overall, TrimerTrip predictions using the AMOEBA force field were consistently the best.

Our in-house reference calculations provided the only other non-ranked submissions for TrimerTrip. Our two sets of reference calculations (Docking/GAFF/YANK_REF and Docking/GAFF/YANK_REF_2) differed only in the choice of host conformer for clipg11, where the latter submission used an alternate, relatively open host conformation to allow it to relax and adapt to the bulky cyclic guest in $\mathrm{g} 11$ (see Section 6.1.1), though this approach ended up not resulting in substantially different predicted binding free energies. Performance statistics for these reference calculations ended up being particularly poor in general (Tables 3 and 4). The reference method gives free energies for all TrimerTrip host-guest complexes which are too unfavorable, similar to ranked MD/DOCKING/GAFF/xtb-GNF predictions. Both submissions used docking (VINA) to obtain guest poses without any MD (except that the MD/DOCKING/GAFF/Xtb-GNF technique used SA-MD to obtain poses for four guests), GAFF parameters, the TIP3P water model, and AM1-BCC charges, so it may not be surprising that performance was similar. However, the MD/DOCKING/GAFF/xtbGNF approach performed better for the case of the four guests where starting poses were established by SA-MD, with errors under $1 \mathrm{kcal} / \mathrm{mol}$ in those cases.

We can perhaps learn a bit more from these non-ranked submissions by comparing to the ranked submission called FSDAM/GAFF2/OPC3, which uses the OPC3 classical 3-point rigid water model with the GAFF2 force field and performed better than methods using its TIP3P counter part with GAFF, though there were other methodological differences between these submissions. The prediction error values for this method were the closest to the top performing methods using the AMOEBA force 


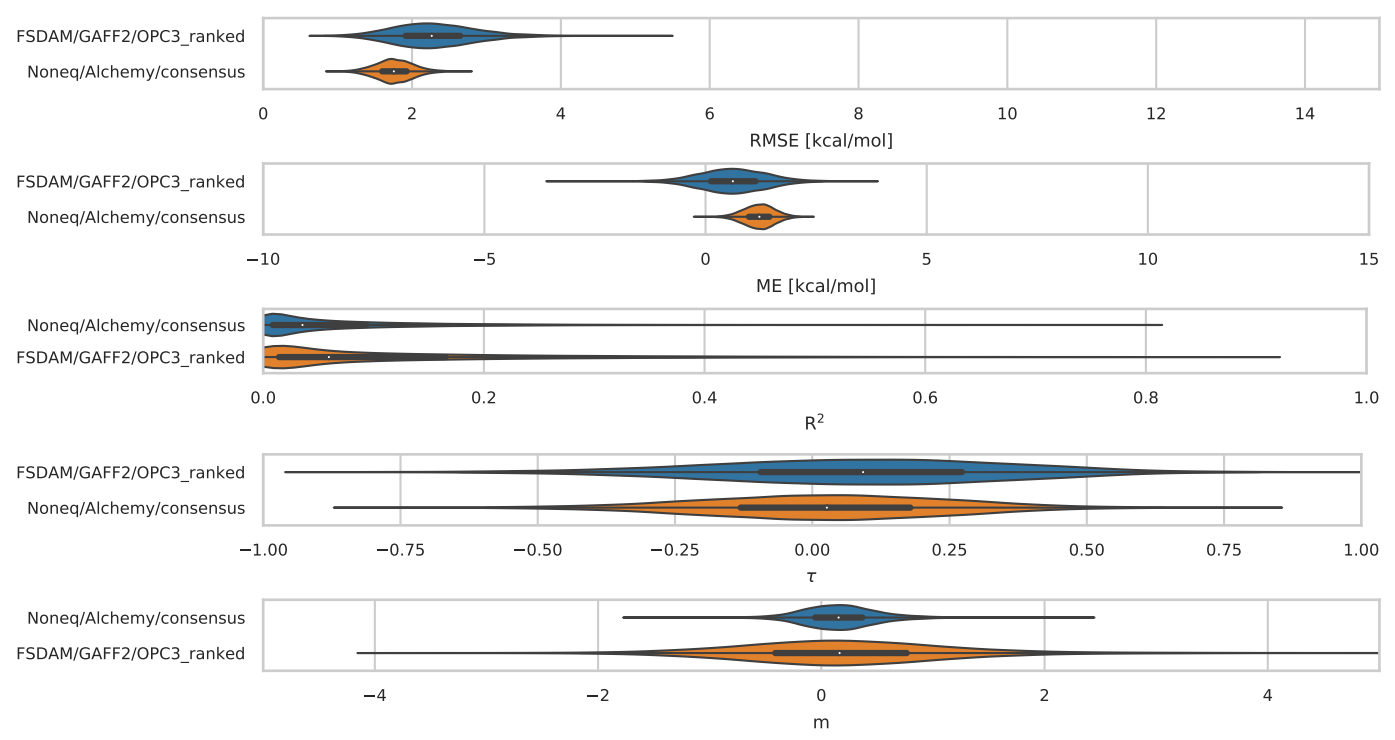

Figure 12. Cyclodextrin derivatives error metrics for ranked methods CD submissions ordered based on the median and is indicated by the white circle in the violin plots. The violin plots were generated by bootstrapping samples with replacement, and the plots describe the shape of the sampling distribution for each prediction. The black horizontal bar represents the first and third quartiles. From top to bottom the error metrics are RMSE, ME, $\mathrm{R}^{2}, \tau$, and slope $(\mathrm{m})$. AM1-BCC/GAFF/TIP4PEW/QMMM method was not included in these plots. In addition, the optional bCD-g1 and bCD-g2 host-guest systems are not included in this analysis.
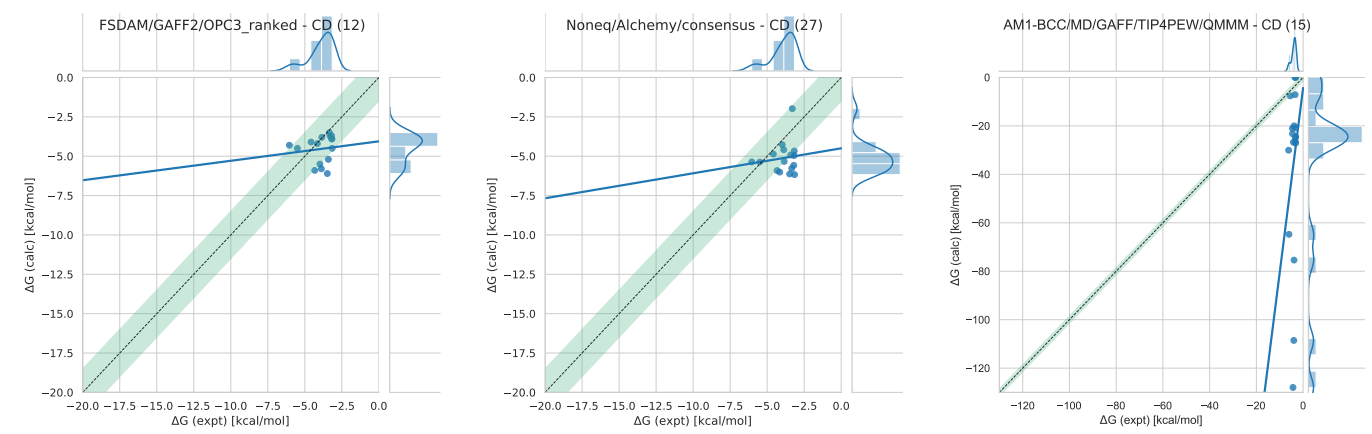

Figure 13. Correlation plots for CD ranked submissions Correlation plots comparing (Left to Right) FSDAM/GAFF2/OPC3, Noneq/Alchemy/consensus, and AM1-BCC/MD/GAFF/TIP4PEW ranked predictions with experimental values for the CD dataset. The $\mathrm{R}^{2}$ and slope for each ranked predictions were 0.04 and $0.17,0.03$ and 0.18 , and 0.04 and 7.62 respectively. Note: the optional bCD-g1 and bCD-g2 host-guest systems were not included in the analysis.

field, however its correlation values were similar to the methods using GAFF/TIP3P. The OPC3 water model has been shown to be significantly more accurate for pure water properties compared to other popular 3-point water models (i.e TIP3P and SPCE) of the same class [89] which may be particularly important in this system. Given the results reported in ref [89] for OPC3 and ref [77] for GAFF2; it is tempting to attribute this method's better performance to use of the OPC3 water model and GAFF2, though without comparison to other methods which differ by only small molecule force field or water model, it is difficult to know this for certain.

\subsubsection{GDCC}

There were 11 non-ranked submissions for the GDCC dataset in addition to the 4 ranked predictions (Table 3). Three of the four participants with ranked submissions included at least two non-ranked submissions which were different in only a single factor, allowing easy sensitivity analysis. For example, all three AMOEBA-based methods had RMSEs below $2 \mathrm{kcal} / \mathrm{mol}$, including the ranked $A M O E B A / D D M / B A R$ submission and the non-ranked AMOEBA/DDM/BAR_2 and AMOEBA/DDM/BAR_3 submissions. These methods differed by key AMOEBA torsional parameters describing the flexibility of the middle and upper rim of the cavity of OA and exo-OA. These differences appear to have substantially affected performance (Table 3). The non-ranked 
Table 3. Error metrics for all (ranked and non-ranked) SAMPL7 methods for all host-guest systems. The root mean square error (RMSE), mean absolute error (MAE), signed mean error (ME), coefficient of correlation $\left(R^{2}\right)$, slope $(\mathrm{m})$, and Kendall's rank correlation coefficient (Tau) were computed via bootstrapping with replacement. Shown are results for individual host categories, as well as the combined $O A$ and exOOA dataset. Statistics do not include optional host-guest systems OA-g1, OA-g2, OA-g3 OA-g4, OA-g5, OA-g6, bCD-g1, and bCD-g2.

\begin{tabular}{|c|c|c|c|c|c|c|c|}
\hline ID & sid & $\operatorname{RMSE}[\mathrm{kcal} / \mathrm{mol}]$ & MAE $[\mathrm{kcal} / \mathrm{mol}]$ & ME [kcal/mol] & $\mathbf{R}^{2}$ & $\mathbf{m}$ & $\tau$ \\
\hline \multicolumn{8}{|l|}{ TrimerTrip } \\
\hline AMOEBA/DDM/BAR/ALT-2 & 9 & $1.58[1.19,2.56]$ & $1.39[0.95,2.23]$ & $-0.36[-1.36,0.68]$ & $0.63[0.18,0.83]$ & $1.14[0.54,1.76]$ & $0.60[0.17,0.80]$ \\
\hline AMOEBAVDDM/BAR-ALT1 & 8 & $1.68[1.28,2.64]$ & $1.56[1.03,2.34]$ & $-0.70[-1.71,0.32]$ & $0.70[0.26,0.88]$ & $1.28[0.70,1.88]$ & $0.67[0.23,0.85]$ \\
\hline AMOEBA/DDM/BAR & 6 & $2.76[1.83,3.98]$ & $2.12[1.35,3.33]$ & $-1.69[-2.98,-0.44]$ & $0.50[0.13,0.77]$ & $1.25[0.53,2.06]$ & $0.47[0.12,0.74]$ \\
\hline FSDAM/GAFF2/OPC3 & 4 & $2.97[2.11,5.13]$ & $2.24[1.62,4.22]$ & $0.43[-1.59,2.33]$ & $0.12[0.00,0.56]$ & $0.60[-0.51,1.60]$ & $0.24[-0.23,0.61]$ \\
\hline MD/DOCKING/GAFF/Xtb-GNF/ & 5 & $5.65[3.87,7.36]$ & $4.51[3.01,6.40]$ & $-4.23[-6.19,-2.23]$ & $0.00[0.00,0.26]$ & $-0.10[-1.02,0.80]$ & $-0.05[-0.41,0.35]$ \\
\hline Docking/GAFF/YANK_REF & REF2 & $7.18[5.63,8.71]$ & $6.57[5.16,8.10]$ & $-6.57[-8.09,-5.16]$ & $0.11[0.00,0.59]$ & $0.57[-0.56,1.55]$ & $0.12[-0.35,0.56]$ \\
\hline Docking/GAFF/YANK_REF_2 & REF3 & $7.21[5.73,8.75]$ & $6.63[5.26,8.13]$ & $-6.63[-8.12,-5.26]$ & $0.12[0.00,0.59]$ & $0.57[-0.55,1.54]$ & $0.12[-0.34,0.57]$ \\
\hline \multicolumn{8}{|l|}{$\overline{\text { GDCC-OA and exoOA }}$} \\
\hline RESP/GAFF/MMPBSA-Cor & 20 & $1.24[0.73,2.45]$ & $0.95[0.57,2.13]$ & $0.94[-0.12,1.99]$ & $0.94[0.10,0.97]$ & $0.65[0.18,1.14]$ & $0.83[0.03,1.00]$ \\
\hline AMOEBA/DDM/BAR & 29 & $1.25[0.68,2.55]$ & $0.92[0.54,2.13]$ & $-0.36[-1.59,0.83]$ & $0.80[0.36,0.97]$ & $1.11[0.58,1.94]$ & $0.72[0.17,1.00]$ \\
\hline AMOEBA/DDM/BAR_2 & 30 & $1.78[0.86,3.24]$ & $1.31[0.67,2.70]$ & $-0.62[-2.09,0.77]$ & $0.55[0.04,0.96]$ & $0.87[0.14,1.92]$ & $0.50[-0.09,1.00]$ \\
\hline xtb-GNF/Machine Learning/CORINA MD & 28 & $2.26[1.38,3.43]$ & $1.91[1.09,3.08]$ & $0.37[-1.27,2.13]$ & $0.01[0.00,0.78]$ & $0.04[-0.58,0.50]$ & $0.06[-0.68,0.78]$ \\
\hline AMOEBA/DDM/BAR_3 3 & 31 & $2.32[1.42,3.58]$ & $2.05[1.13,3.22]$ & $-0.29[-1.95,1.52]$ & $0.61[0.21,0.92]$ & $1.30[0.54,2.41]$ & $0.78[0.24,1.00]$ \\
\hline Docking/GAFF/YANK_REF & REF4 & $4.05[1.54,5.88]$ & $2.90[1.21,4.93]$ & $2.40[0.41,4.67]$ & $0.12[0.00,0.65]$ & $-0.30[-1.06,0.53]$ & $-0.11[-0.70,0.60]$ \\
\hline B2PLYPD3/SMD_QZ-R & 23 & $4.52[2.55,6.41]$ & $3.70[1.95,5.69]$ & $3.15[0.85,5.50]$ & $0.49[0.02,0.92]$ & $1.43[-0.17,2.92]$ & $0.37[-0.33,0.88]$ \\
\hline B2PLYPD3/SMD_QZ-NR & 24 & $4.64[2.77,6.46]$ & $3.95[2.23,5.83]$ & $2.69[0.06,5.33]$ & $0.58[0.03,0.96]$ & $1.84[-0.24,3.28]$ & $0.39[-0.31,0.93]$ \\
\hline FSDAM/GAFF2/OPC3 & 14 & $5.07[3.12,8.84]$ & $4.69[2.53,7.86]$ & $-0.79[-5.32,3.40]$ & $0.77[0.01,0.94]$ & $-1.26[-2.65,0.18]$ & $-0.59[-1.00,0.24]$ \\
\hline B2PLYPD3/SMD_TZ & 22 & $5.08[3.04,7.03]$ & $4.22[2.39,6.37]$ & $3.36[0.67,6.04]$ & $0.58[0.02,0.96]$ & $1.85[-0.29,3.31]$ & $0.39[-0.33,0.94]$ \\
\hline RESP/GAFF/MMPBSA/Nmode & 18 & $5.84[4.47,7.31]$ & $5.60[4.20,7.03]$ & $-5.60[-7.03,-4.20]$ & $0.81[0.44,0.98]$ & $1.40[0.79,2.40]$ & $0.83[0.31,1.00]$ \\
\hline RESP/GAFF/MMPBSA & 19 & $8.07[6.96,9.33]$ & $7.98[6.81,9.20]$ & $7.98[6.81,9.20]$ & $0.94[0.54,0.99]$ & $1.45[0.96,1.99]$ & $0.83[0.39,1.00]$ \\
\hline B2PLYPD3/SMD_DZ & 21 & $8.13[5.62,10.34]$ & $7.17[4.57,9.75]$ & $7.17[4.48,9.75]$ & $0.55[0.02,0.96]$ & $1.80[-0.36,3.28]$ & $0.39[-0.33,0.94]$ \\
\hline AM1-BCC/GAFF/MMPBSA & 17 & $10.96[9.02,12.80]$ & $10.61[8.59,12.59]$ & $10.61[8.59,12.59]$ & $0.91[0.59,0.99]$ & $2.12[1.55,2.83]$ & $0.89[0.43,1.00]$ \\
\hline RESP/GAFF/MMGBSA & 16 & $11.85[10.29,13.47]$ & $11.68[10.12,13.29]$ & $11.68[10.12,13.29]$ & $0.88[0.40,0.99]$ & $1.69[1.10,2.36]$ & $0.78[0.23,1.00]$ \\
\hline \multicolumn{8}{|l|}{$\overline{\text { GDCC - exoOA }}$} \\
\hline AMOEBA/DDM/BAR_2 & 30 & $1.23[0.65,2.53]$ & $1.02[0.51,2.25]$ & $-0.13[-1.47,1.27]$ & $0.83[0.39,0.99]$ & $1.21[0.56,2.29]$ & $0.62[0.16,1.00]$ \\
\hline AMOEBA/DDM/BAR & 29 & $1.27[0.56,2.72]$ & $0.91[0.45,2.31]$ & $-0.66[-1.98,0.61]$ & $0.81[0.30,0.99]$ & $1.05[0.45,2.12]$ & $0.71[0.05,1.00]$ \\
\hline RESP/GAFF/MMPBSA-Cor & 20 & $1.32[0.68,2.65]$ & $1.03[0.54,2.34]$ & $1.01[-0.18,2.20]$ & $0.95[0.04,0.99]$ & $0.61[0.04,1.20]$ & $0.81[-0.14,1.00]$ \\
\hline AMOEBA/DDM/BAR_3 & 31 & $1.72[0.93,3.04]$ & $1.57[0.75,2.77]$ & $-1.44[-2.66,-0.19]$ & $0.79[0.15,0.99]$ & $0.80[0.22,1.72]$ & $0.81[-0.05,1.00]$ \\
\hline xtb-GNF/Machine Learning/CORINA MD & 28 & $2.43[1.40,3.71]$ & $2.11[1.10,3.42]$ & $0.82[-1.12,2.77]$ & $0.00[0.00,0.91]$ & $0.01[-0.81,0.57]$ & $0.05[-0.78,1.00]$ \\
\hline Docking/GAFF/YANK_REF & REF4 & $4.48[1.56,6.43]$ & $3.25[1.10,5.65]$ & $2.60[0.06,5.40]$ & $0.37[0.03,0.95]$ & $-0.58[-1.56,0.08]$ & $-0.43[-1.00,0.33]$ \\
\hline B2PLYPD3/SMD_QZ-R & 23 & $4.76[2.26,6.93]$ & $3.90[1.81,6.26]$ & $3.50[0.91,6.12]$ & $0.72[0.24,0.99]$ & $1.97[0.88,3.77]$ & $0.59[-0.06,1.00]$ \\
\hline FSDAM/GAFF2/OPC3 & 14 & $4.85[2.61,8.41]$ & $4.38[2.13,7.58]$ & $0.62[-3.93,5.08]$ & $0.82[0.01,0.99]$ & $-1.24[-2.89,0.30]$ & $-0.59[-1.00,0.33]$ \\
\hline B2PLYPD3/SMD_QZ-NR & 24 & $4.90[2.64,6.93]$ & $4.23[2.23,6.33]$ & $2.91[-0.26,5.90]$ & $0.80[0.26,0.99]$ & $2.46[0.99,3.87]$ & $0.62[0.00,1.00]$ \\
\hline B2PLYPD3/SMD_TZ & 22 & $5.36[2.93,7.56]$ & $4.57[2.40,6.98]$ & $3.60[0.40,6.66]$ & $0.81[0.24,0.99]$ & $2.48[0.90,3.84]$ & $0.62[-0.05,1.00]$ \\
\hline RESP/GAFF/MMPBSA/Nmode & 18 & $6.28[4.78,7.92]$ & $6.09[4.54,7.71]$ & $-6.09[-7.71,-4.54]$ & $0.76[0.26,0.99]$ & $1.26[0.47,2.43]$ & $0.81[0.11,1.00]$ \\
\hline RESP/GAFF/MMPBSA & 19 & $7.59[6.37,8.90]$ & $7.53[6.25,8.79]$ & $7.53[6.25,8.79]$ & $0.95[0.48,1.00]$ & $1.36[0.74,1.96]$ & $0.81[0.29,1.00]$ \\
\hline B2PLYPD3/SMD_DZ & 21 & $8.41[5.40,10.95]$ & $7.42[4.31,10.43]$ & $7.42[4.23,10.42]$ & $0.79[0.22,0.99]$ & $2.44[0.84,3.81]$ & $0.62[0.00,1.00]$ \\
\hline AM1-BCC/GAFF/MMPBSA & 17 & $10.05[7.92,12.08]$ & $9.73[7.58,11.84]$ & $9.73[7.58,11.84]$ & $0.93[0.61,1.00]$ & $2.06[1.38,2.94]$ & $0.90[0.29,1.00]$ \\
\hline RESP/GAFF/MMGBSA & 16 & $11.11[9.58,12.68]$ & $11.00[9.46,12.56]$ & $11.00[9.46,12.56]$ & $0.96[0.66,1.00]$ & $1.67[1.09,2.38]$ & $0.90[0.37,1.00]$ \\
\hline \multicolumn{8}{|l|}{ Cyclodextrin derivatives } \\
\hline FSDAM/GAFF2/OPC__ranked & 12 & $1.23[1.36,3.39]$ & $1.01[1.06,2.84]$ & $0.47[-0.90,1.87]$ & $0.04[0.00,0.46]$ & $0.17[-1.26,1.66]$ & $0.23[-0.41,0.55]$ \\
\hline Noneq/Alchemy/CGENFF & 26 & $1.55[1.17,2.33]$ & $1.35[0.93,2.03]$ & $0.99[0.24,1.74]$ & $0.05[0.00,0.39]$ & $0.24[-0.45,0.95]$ & $0.10[-0.41,0.49]$ \\
\hline Noneq/Alchemy/consensus & 27 & $1.62[1.21,2.17]$ & $1.38[0.96,1.90]$ & $1.08[0.43,1.72]$ & $0.03[0.00,0.30]$ & $0.18[-0.33,0.74]$ & $0.03[-0.38,0.45]$ \\
\hline FSDAM/GAFF2/OPC3JB & 13 & $1.71[1.55,3.76]$ & $1.48[1.21,3.19]$ & $0.54[-0.94,2.04]$ & $0.01[0.00,0.41]$ & $-0.14[-1.58,1.47]$ & $0.03[-0.44,0.48]$ \\
\hline Noneq/Alchemy/GAFF & 25 & $1.84[1.35,2.58]$ & $1.54[1.07,2.24]$ & $1.17[0.37,1.97]$ & $0.01[0.00,0.28]$ & $0.12[-0.55,0.83]$ & $0.02[-0.36,0.43]$ \\
\hline Docking/GAFF/YANK_REF & REF1 & $2.64[1.87,3.42]$ & $2.19[1.51,2.94]$ & $0.64[-0.58,1.84]$ & $0.02[0.00,0.36]$ & $-0.29[-1.59,0.87]$ & $-0.10[-0.44,0.24]$ \\
\hline AM1-BCC/MD/GAFF/TIPUPEW/QMMM & 15 & $46.62[22.85,65.69]$ & $32.00[17.92,49.22]$ & $31.27[16.89,48.87]$ & $0.04[0.00,0.33]$ & $7.62[-3.31,30.72]$ & $0.24[-0.13,0.52]$ \\
\hline
\end{tabular}

AMOEBA/DDM/BAR_2 RMSE was the best of all methods and all predicted binding free energies were within $2 \mathrm{kcal} / \mathrm{mol}$ of the experimental values, including those for exoOA-g4, which was poorly predicted by other AMOEBA methods. The host parameters used in AMOEBA/DDM/BAR_2 were similar to those used in previous SAMPL challenges, while the other predictions used modified parameters. Overall, these AMOEBA submissions suggest guest binding to GDCCs is particularly sensitive to the host's diphenyl ether torsions, and especially so for guest $\mathrm{g} 4$ binding to exo-OA and guests $\mathrm{g} 7$ and g8 binding to OA.

Another ranked submission which performed well used MM/PBSA, and non-ranked variants of this explored variations based on both MM/PBSA and MM/GBSA. One variation assessed the charge model, and found that the RESP charge scheme led to improved performance compared to the AM1-BCC charge scheme (RESP/GAFF/MMPBSA vs AM1-BCC/GAFF/MMPBSA), as shown by RMS errors of $7.59 \mathrm{kcal} / \mathrm{mol}$ vs $10.96 \mathrm{kcal} / \mathrm{mol}$. These methods predicted binding free energies to be too favorable, a common issue with such endpoint free energy methods, especially when entropy changes are neglected, as here. An additional variation assessed the difference between MM/PBSA and MM/GBSA by changing the solvent model; the use of PB solvation resulted in significantly lower RMS errors here (RESP/GAFF/MMPBSA vs RESP/GAFF/MMGBSA), though the correlation with the GB approach was modestly better. A further variation added an accounting for entropy via normal mode analysis (RESP/GAFF/MMPBSA/Nmode) while maintaining RESP charges and PB solvation. This improved typical errors, but hurt correlation and resulted in binding free energies often not being favorable enough. One other key difference between the ranked submission in this series, and many of the others was that it actually used an empirical correction to binding free energies. Particularly, RESP/GAFF/MMPBSA-Cor 
used a linear correction derived from an analysis of previous SAMPL challenges [43] . Indeed, this correction led to much better agreement with experiments. With RMSE and ME values of 1.32 and $1.01 \mathrm{kcal} / \mathrm{mol}$, the RESP/GAFF/MMPBSA-Cor performance was on par with AMOEBA results, and for some guests performed even slightly better. In terms of correlation, the ranked RESP/GAFF/MMPBSA-Cor was similar to that of RESP/GAFF/MMPBSA. However, such an approach could not be applied without prior binding studies for the specific system(s) of interest.

A series of density functional theory (DFT)-based methods, including ranked and non-ranked submissions, were also used here. In SAMPL6, a DFT-based approach yielded good qualitative results $[4,5,43]$, though without geometry optimizations employed in the current challenge. Here, the QM DFT-based B2PLYPD3/SMD submissions use B3PW91 with GD3BJ [2] to treat dispersion, B2PLYPD3 for single point energy calculations [1], and the SMD implicit solvation model [90] Different submissions in this series differed in which basis set was chosen for geometry optimization. Overall, these methods were roughly in the middle of all submitted methods in terms of predictive accuracy. All of these QM methods yield binding free energies for most guests which are too negative, with ME values of $2.69 \mathrm{kcal} / \mathrm{mol}$ or greater, and this is especially true for cationic guests binding exo$\mathrm{OA}$. The participants also highlighted particular difficulties with chlorine-containing guest g2. In initial tests, the OA-g2 binding free energy was estimated to be close to $-30 \mathrm{kcal} / \mathrm{mol}$, while experimental value in literature was $-6.91 \mathrm{kcal} / \mathrm{mol}$ [43]. In the combined GDCC dataset, the ranked B2PLYPD3/SMD_QZ-R method was within $2 \mathrm{kcal} / \mathrm{mol}$ of experiments in three of nine cases and correctly predicted exoOA-g1 to be a non-binder. Overall, it appears that QM methods are not yet competitive with the best other approaches for these systems, and potentially, that halogens can be particularly problematic.

Two of the non-ranked methods do not allow for straightforward sensitivity analysis based on a single factor, because only a single version was submitted (FSDAM/GAFF2/OPC3 and reference calculation DOCKING/GAFF/YANK). Both of these methods were also used for TrimerTrip and the cyclodextrin challenge. The error metrics for both of the methods were relatively similar, although the DOCKING/GAFF/YANK method performed slightly better by a number of metrics. However, the ME for FS$D A M / G A F F 2 / O P C 3$ is quite low - less than $1 \mathrm{kcal} / \mathrm{mol}$ - because the method tends to predict binding free energies for OctaAcids with guests bearing a carboxylate group which are too favorable, and too unfavorable for guests with cationic ammoniums. In comparison, DOCKING/GAFF/YANK errs for all guests with carboxylate group are too favorable. Still, enough things differ between these two submissions that it is difficult to attribute performance differences to any particular source. Such simple variations provide the greatest opportunity for the community to learn.

One exoOA guest posed a bit of a surprise, in that binding of $\mathrm{g} 1$ to exoOA was not detected experimentally (Section 6.2 and Figure 1). Of the 15 GDCC submissions, 7 predicted this correctly with computed free energies ranging between -0.98 and $6.40 \mathrm{kcal} / \mathrm{mol}$. Of the methods which incorrectly predicted g1 to bind, computed binding free energies ranged from -2.45 to $-11.54 \mathrm{kcal} / \mathrm{mol}$. All of the QM based submissions (B2PLYPD3/SMD) predicted exoOA-g1 to be a nonbinder, with values between 2.70 and $6.40 \mathrm{kcal} / \mathrm{mol}$, and the AMOEBA-based methods also correctly recognized this as a nonbinder. Most MM/PBSA and MM/GBSA submissions failed to recognize this as a nonbinder, except for the RESP/GAFF/MMPBSA/Nmode method utilizing empirical corrections. The other GAFF-based methods predicted exoOA-g1 to be a binder. Predicted binding free energies of xtb-GNF/MachineLearning/CORINA_MD, RESP/GAFF/MMPBSA-Cor, RESP/GAFF/MMGBSA, AM1-BCC/GAFF/MMPBSA, RESP/GAFF/MMPBSA, FSDAM/GAFF2/OPC3, and DOCKING/GAFF/YANK were all more favorable than $-3.84 \mathrm{kcal} / \mathrm{mol}$. Perhaps for this guest, the proximal carboxylates of the host and guest repel one another too strongly for binding. This guest has relatively less hydrophobic character than other guests, perhaps meaning that the hydrophobic effect is not enough to offset this potential electrostatic clash. Perhaps only the AMOEBA and the QM based methods can capture the relevant polarization effects well enough to recognize this complex is unfavorable.

\subsubsection{Cyclodextrins}

The cyclodextrin challenge proved to be the least challenging of the SAMPL7 challenges as measured by RMS error, as all submissions except one had RMSE values less than $2.74 \mathrm{kcal} / \mathrm{mol}$ (the exception was the AM1-BCC/MD/GAFF/TIP4PEW/QMMM method,with RMSE and ME metrics over $30 \mathrm{kcal} / \mathrm{mol}$ ). However, the dynamic range was particularly small for this challenge, with most host-guest complexes showing similar binding free energies. This meant that correlations between calculated and predicted values were typically quite poor (Table 3).

First we compare the ranked FSDAM/GAFF2/OPC3_ranked and non-ranked FSDAM/GAFF2/OPC3JB methods, where the ranked method performed slightly better; these methods used the same simulation approach but differ in that the former used a Gaussian approximation for computing nonequilibrium free energies, whereas the latter used a "boosted Jarzynsi" approach for analysis [88]. Both analysis approaches ought to give equivalent binding free energies in certain limits, but their underlying assumptions and the amount of data available result in substantially different performance here. Here, despite its limitations in 
the SAMPL6 "SAMPLing" challenge [5], the Gaussian approximation was modestly superior, with, 15 of 16 binding free energies predicted within $2 \mathrm{kcal} / \mathrm{mol}$, versus 11 of 16 for FSDAM/GAFF2/OPC3JB.

Three other nonequilibrium free energy methods participated for the cyclodextrin challenge - Noneq/Alchemy/CGENFF, Noneq/Alchemy/cc and Noneq/Alchemy/GAFF. All three methods used the TIP3P water model, included NaCl ions at $25 \mathrm{mM}$, and considered multiple binding poses (primary and secondary orientation) and free energies reported as Boltzmann weighted averages across these poses. These methods differed only by force field - CGENFF (Noneq/Alchemy/CGENFF) or GAFF (Noneq/Alchemy/GAFF). The third submission, Noneq/Alchemy/consensus, gives "consensus" results obtained by averaging across both force fields. In this case the RMSE was under $2 \mathrm{kcal} / \mathrm{mol}$ for both methods, but CGENFF resulted in very slightly better performance by most metrics. Problematic systems for this method were MGLab23-g1, MGLab24-g1, MGLab24g2, MGLab36-g1, and MGLab36-g2, and what they have in common is larger cyclodextrin side chains. Cyclodextrins with amino acid side chains tend to be the more accurately predicted systems for this method, suggesting the methods may be limited by forcefield parameters.

Our reference calculations (Docking/GAFF/YANK_REF performed reasonably well for this dataset (Table 4), and surprisingly had better correlation to experiments compared to other methods (Table 3). Predicted binding free energies were within 2 $\mathrm{kcal} / \mathrm{mol}$ for 9 of 16 host-guest systems, and similar conditions and water model were used as for Noneq/Alchemy/GAFF, though different free energy estimation techniques were used. These submissions also differed in handling of binding modes; our reference calculations used only a single initial binding mode for each (determined by the top scoring pose from docking) whereas Noneq/Alchemy/consensus considered up to two poses whenever a second orientation was considered stable and was in better agreement with experiments. Thus, suggesting secondary guests orientations may need to be considered, and guest and/or host side chain sampling may be an issue.

When we compare the diverse methods submitted, some observations stand out. First, performance of Noneq/Alchemy/GAFF and Docking/GAFF/YANK_REF methods was quite similar - likely due to use of the same force field (GAFF) and water model (TIP3P) despite the fact that the former used nonequilibrium free energy techniques and the latter used equilibrium, suggesting the force field played a larger role. Along the same lines, several nonequilibrium methods (FSDAM/GAFF2/OPC3, Noneq/Alchemy/CGENFF, and Noneq/Alchemy/GAFF) all used similar techniques but different force field/water model, and performance was thus quite varied. In addition, binding free energy calculations have been shown to be more accurate using GAFF2 opposed to GAFF in previous comptuational studies [77]. Finally, the most challenging case seems to be binding of cyclodextrins with large sidechains to rimantadine (g2), though the exact reason for this is not known.

Table 4. Error metrics for methods used in reference binding free energy calculations of all hostguest systems Please see section 6.1.1 for details on the submission methodology. Optional systems in the GDCC and cyclodextrin datasets (OA-g1, OA-g2, OA-g3, OA-g4, OA-g5, OA-g6, bCD-g1, and bCD-g2) are not part of this analysis. This table includes the method ID, method submission ID (sid), root mean squared error (RMSE), mean absolute error (MAE), mean signed error (ME), coefficient of determination $\left(\mathrm{R}^{2}\right)$, linear regression slope $(\mathrm{m})$, and kendall rank correlation coefficient $(\tau)$ for cyclodextrin, TrimerTrip, combined GDCC dataset (includes both OA and exoOA predictions), and exoOA datasets.

\begin{tabular}{|c|c|c|c|c|c|c|c|}
\hline ID & sid & RMSE [kcal/mol] & MAE [kcal/mol] & ME [kcal/mol] & $\mathbf{R}^{2}$ & m & $\tau$ \\
\hline \multicolumn{8}{|l|}{ Cyclodextrin derivatives } \\
\hline Docking/GAFF/YANK_REF & REF1 & $2.64[1.87,3.42]$ & $2.19[1.51,2.94]$ & $0.64[-0.58,1.84]$ & $0.02[0.00,0.36]$ & $-0.29[-1.59,0.87]$ & $-0.10[-0.44,0.24]$ \\
\hline \multicolumn{8}{|l|}{ TrimerTrip } \\
\hline Docking/GAFF/YANK_REF & REF2 & $7.18[5.63,8.71]$ & $6.57[5.16,8.10]$ & $-6.57[-8.09,-5.16]$ & $0.11[0.00,0.59]$ & $0.57[-0.56,1.55]$ & $0.12[-0.35,0.56]$ \\
\hline Docking/GAFF/YANK_REF_2 & REF3 & $7.21[5.73,8.75]$ & $6.63[5.26,8.13]$ & $-6.63[-8.12,-5.26]$ & $0.12[0.00,0.59]$ & $0.57[-0.55,1.54]$ & $0.12[-0.34,0.57]$ \\
\hline \multicolumn{8}{|l|}{$\overline{\text { GDCC }-O A \text { and exoOA }}$} \\
\hline Docking/GAFF/YANK_REF & REF4 & $4.05[1.54,5.88]$ & $2.90[1.21,4.93]$ & $2.40[0.41,4.67]$ & $0.12[0.00,0.65]$ & $-0.30[-1.06,0.53]$ & $-0.11[-0.70,0.60]$ \\
\hline \multicolumn{8}{|l|}{$\overline{\text { GDCC - exoOA }}$} \\
\hline Docking/GAFF/YANK_REF & REF4 & $4.48[1.56,6.43]$ & $3.25[1.10,5.65]$ & $2.60[0.06,5.40]$ & $0.37[0.03,0.95]$ & $-0.58[-1.56,0.08]$ & $-0.43[-1.00,0.33]$ \\
\hline
\end{tabular}

\section{Conclusions and Lessons Learned}

The SAMPL7 host-guest blind challenge provided a platform to test the reliability of computational methods and tools to accurately predict binding free energies. Since hosts in the cucurbituril and cavitand families have been featured in previous SAMPL challenges (and likely in future challenges) these provide a mechanism to assess how the field progresses across a series of challenges. In addition, the amount of attention these have received helps us identify some potential lessons learned and give suggestions for improvement. 
The TrimerTrip dataset of SAMPL7, like cucurbiturils from previous challenges, posed the largest challenge for participants, as judged by method performance. Specifically, most methods performed poorly at computing binding free energies for cationic guests with cyclic, aromatic, and adamantane based moieties. In addition, most methods were relatively inconsistent at predicting binding free energies of hydrocarbon chains of increasing length, but the AMOEBA methods did very well predicting 7 of 8 within $2 \mathrm{kcal} / \mathrm{mol}$. Still, two methods performed relatively well even here, with both using alchemical free energy calculations. Predictions from the best fixed-charge force field submission, based on nonequilibrium free energy calculations (FSDAM/GAFF2/OPC3), had errors above $2 \mathrm{kcal} / \mathrm{mol}$ for $8 / 16$ host-guest systems considered. In contrast, performance with the AMOEBA polarizable force field was significantly better here, suggesting that one key source of error may be polarization effects.

In the TrimerTrip case, participants also found evidence that binding free energies may be more accurate if different potential host conformations are considered, especially for bulkier guests such as those with adamantane moeities. This exploration of sensitivity to host conformation also provided insight into modeling the the host's flexibility; participants found binding free energies to be sensitive to the torsional parameters of the glycoluril methylene bridges [43].Our reference calculations showed poor sampling of interconversion between alchemical states in our simulations, despite use of Hamiltonian Replica Exchange.

Given these results, it appears that force field accuracy and choice of force field (e.g. GAFF, GAFF2, AMOEBA) may be a dominant factor limiting accurate binding affinity predictions.

On the Gibb deep cavity cavitands (GDCCS), OA and exoOA, as in previous SAMPL challenges, simulation based methods with empirical fixed charge energy models performed relatively well. Binding affinities for guests with adamantane, aromatic and saturated cyclic carboxlylates with OctaAcids were predicted with greater accuracy than TrimerTrip. Performance of methods within the GDCC dataset (OA and exOOA) demonstrates significant variation by guest, and especially when the formal charge of guest differs (negative vs positive).

In part because of the relatively extensive prior work on GDCCs, some submissions applied empirical corrections before making predictions, and/or utilized machine learning approaches. These tended to help performance, here, but rely on availability of training data on closely related systems - which is not always available for prospective applications.

On the GDCCs, as for TrimerTrip, submissions using the AMOEBA force field performed particularly well. Additionally, along with a QM based method, AMOEBA correctly predicted exOOA with g1 a non-bonder. Perhaps only AMOEBA and QM methods capture relevant polarization effects well enough to accurately describe this particular complex well in general, though one MM/PBSA approach also recognized this as a nonbinder.

For the current challenge, the AMOEBA method had the most consistent performance across the different host-guest complexes, and across datasets (TrimerTrip, OctaAcid, exoOA). Despite the lower variation for this method, guest g4 was particularly sensitive to diphenyl ether torsional parameters which worked very well in all other GDCC systems.

The cyclodextrin derivatives were new to SAMPL, and many methods achieved relatively low RMS errors - though this is likely in part due to the low dynamic range of the set. This low dynamic range also meant that correlation metrics were typically poor. There were no AMOEBA submissions for this aspect of SAMPL7, but the force field used in this dataset still apparently played a role computing accurate binding free energies, with GAFF2 seemingly giving more accurate results followed by CGenFF, and GAFF. The performance of methods for the cyclodextrin dataset varied across host-guest systems, but predicting reliable binding free energies for cylcodextrins with large sidechains to rimantadine was frequently challenging.

Finally, note that two methods included predictions for all three datasets, DOCKING/GAFF/YANK and FSDAM/GAFF2/OPC, though not all of the submissions were ranked. The performance of these methods varied across different datasets and across different host-guest systems within the same dataset. For both methods, binding predictions for larger and more hydrophobic guests were apparently more difficult.

In terms of overall lessons learned in this challenge, we found that methods which only varied a single factor (such as force field or water model, with a fixed method) were particularly valuable in terms of providing insight into accuracy, thus we urge participants to continue with such explorations in the future. Another important area of work is to ensure that methods which ought to be equivalent do, in fact, give equivalent results across different simulation packages. [5].

Overall, SAMPL7 showed marked progress in binding prediction relative to previous challenges, and in particular results with the AMOEBA force field were particularly promising for two of the challenge components. For future challenges it will be interesting to continue investigations of host/guest sampling, polarization effects, and possibly salt behavior in similar systems. We look forward to continuing to work with the community to use the SAMPL challenge to drive accuracy improvements in binding predictions. 
Table 5. Methods used as reference calculations for the MM-based physical methods category.

\begin{tabular}{llllll}
\hline Submission ID & Approach & Force Field & Water Model & Octanol Phase & Number of Replicates \\
\hline REF01 & YANK, DFE protocol & GAFF 1.81 & TIP3P-FB & Wet & 3 \\
REF02 & YANK, DFE protocol & GAFF 1.81 & TIP3P & Wet & 3 \\
REF03 & YANK, DFE protocol & GAFF 1.81 & OPC & Wet & 3 \\
REF04 & YANK, DFE protocol & smirnoff99Frosst 1.0.7 & TIP3P-FB & Wet & 3 \\
REF05 & YANK, DFE protocol & smirnoff99Frosst 1.0.7 & TIP3P & Wet & 3 \\
REF06 & YANK, DFE protocol & smirnoff99Frosst 1.0.7 & OPC & Wet & 3 \\
REF07 & YANK, DFE protocol & GAFF 1.81 & TIP3P & Dry & 3 \\
REF08 & YANK, DFE protocol & smirnoff99Frosst 1.0.7 & TIP3P & Dry & 3 \\
\hline
\end{tabular}

\section{Code and Data Availability}

All SAMPL7 host-guest challenge instructions, submissions, experimental data and analysis are available at https://github.com/samplchallenges/SAMPL7/tree/master/host_guest. An archive copy of SAMPL7 GitHub repository host-guest challenge directory is also available in the Supplementary Documents bundle (SAMPL7-supplementary-documents.tar.gz). Some useful files from this repository are highlighted below.

- Table of participants submission filenames and their submission ID:

https://github.com/samplchallenges/SAMPL7/blob/master/host guest/Analysis/SAMPL7-user-map-HG.csv

- Submission files of prediction sets:

https://github.com/samplchallenges/SAMPL7/tree/master/host_guest/Analysis/Submissions

- Python analysis scripts and outputs:

https://github.com/samplchallenges/SAMPL7/tree/master/host_guest/Analysis/Scripts

- Table of performance statistics calculated for ranked methods for TrimerTrip dataset:

https://github.com/samplchallenges/SAMPL7/blob/master/host_guest/Analysis/Accuracy_ranked/TrimerTrip/StatisticsTables/statistics. csv

- Table of performance statistics calculated for all methods for TrimerTrip dataset:

https://github.com/samplchallenges/SAMPL7/blob/master/host guest/Analysis/Reference/Accuracy/TrimerTrip/StatisticsTables/ statistics.csv

- Table of performance statistics calculated for ranked methods for GDCC dataset:

https://github.com/samplchallenges/SAMPL7/blob/master/host_guest/Analysis/Accuracy_ranked/GDCC_no_optional/StatisticsTables/ statistics.csv

- Table of performance statistics calculated for all methods for GDCC (without optionals) dataset:

https://github.com/samplchallenges/SAMPL7/blob/master/host_guest/Analysis/Reference/Accuracy/GDCC_no_optional/StatisticsTables/ statistics.csv

- Table of performance statistics calculated for all methods for GDCC (with optionals) dataset:

https://github.com/samplchallenges/SAMPL7/blob/master/host guest/Analysis/Reference/Accuracy/GDDC/StatisticsTables/statistics. csv

- Table of performance statistics calculated for ranked methods for Cyclodextrin dataset:

https:/github.com/samplchallenges/SAMPL7/blob/master/host guest/Analysis/Accuracy ranked/CD no optional/StatisticsTables/ statistics.csv

- Table of performance statistics calculated for all methods for Cyclodextrin (without optionals) dataset:

https://github.com/samplchallenges/SAMPL7/blob/master/host_guest/Analysis/Reference/Accuracy/CD_no_optional/StatisticsTables/ statistics.csv

- Talbe of performance statistics calculated for all methods for Cyclodextrin (with optionals) dataset: https://github.com/samplchallenges/SAMPL7/blob/master/host_guest/Analysis/Reference/Accuracy/CD/StatisticsTables/statistics. csv 


\section{Acknowledgments}

MA and DLM gratefully acknowledge support from NIH grant R01GM124270 supporting the SAMPL Blind Challenges. We appreciate the laboratories of Michael K. Gilson (UCSD), Lyle Isaacs (Maryland) and Bruce Gibb (Tulane) for providing experimental data for the challenge. We are also grateful to OpenEye Scientific for providing a free academic software license for use in this work.

\section{Disclaimers}

The content is solely the responsibility of the authors and does not necessarily represent the official views of the National Institutes of Health.

\section{Disclosures}

DLM is a member of the Scientific Advisory Board of OpenEye Scientific Software, and DLM is an Open Science Fellow with Silicon Therapeutics.

\section{References}

[1] Goerigk L, Grimme S. Efficient and Accurate Double-Hybrid-Meta-GGA Density Functionals-Evaluation with the Extended GMTKN30 Database for General Main Group Thermochemistry, Kinetics, and Noncovalent Interactions. Journal of chemical theory and computation. 2011; doi: 10.1021/ct100466k.

[2] Grimme S, Ehrlich S, Goerigk L. Effect of the Damping Function in Dispersion Corrected Density Functional Theory. J Comput Chem. 2011 May; 32(7):1456-1465. doi: 10.1002/jcc.21759.

[3] Yin J, Henriksen NM, Slochower DR, Shirts MR, Chiu MW, Mobley DL, Gilson MK. Overview of the SAMPL5 Host-Guest Challenge: Are We Doing Better? J Comput Aided Mol Des. 2017 Jan; 31(1):1-19. doi: 10.1007/s10822-016-9974-4.

[4] Rizzi A, Murkli S, McNeill JN, Yao W, Sullivan M, Gilson MK, Chiu MW, Isaacs L, Gibb BC, Mobley DL, Chodera JD. Overview of the SAMPL6 Host-Guest Binding Affinity Prediction Challenge. J Comput Aided Mol Des. 2018 Oct; 32(10):937-963. doi: 10.1007/s10822-018-0170-6.

[5] Rizzi A, Jensen T, Slochower DR, Aldeghi M, Gapsys V, Ntekoumes D, Bosisio S, Papadourakis M, Henriksen NM, de Groot BL, Cournia Z, Dickson A, Michel J, Gilson MK, Shirts MR, Mobley DL, Chodera JD. The SAMPL6 SAMPLing Challenge: Assessing the Reliability and Efficiency of Binding Free Energy Calculations. J Comput Aided Mol Des. 2020 Jan; doi: 10.1007/s10822-020-00290-5.

[6] Wang L, Wu Y, Deng Y, Kim B, Pierce L, Krilov G, Lupyan D, Robinson S, Dahlgren MK, Greenwood J, Romero DL, Masse C, Knight JL, Steinbrecher T, Beuming T, Damm W, Harder E, Sherman W, Brewer M, Wester R, et al. Accurate and Reliable Prediction of Relative Ligand Binding Potency in Prospective Drug Discovery by Way of a Modern Free-Energy Calculation Protocol and Force Field. J Am Chem Soc. 2015 Feb; 137(7):2695-2703. doi: 10.1021/ja512751q.

[7] Cournia Z, Allen B, Sherman W. Relative Binding Free Energy Calculations in Drug Discovery: Recent Advances and Practical Considerations. J Chem Inf Model. 2017 Dec; 57(12):2911-2937. doi: 10.1021/acs.jcim.7b00564.

[8] Rocklin G], Mobley DL, Dill KA, Hünenberger PH. Calculating the Binding Free Energies of Charged Species Based on Explicit-Solvent Simulations Employing Lattice-Sum Methods: An Accurate Correction Scheme for Electrostatic Finite-Size Effects. J Chem Phys. 2013 Nov; 139(18):184103. doi: 10.1063/1.4826261.

[9] Mobley DL, Gilson MK. Predicting Binding Free Energies: Frontiers and Benchmarks. Annu Rev Biophys. 2017 May; 46(1):531-558. doi: 10.1146/annurev-biophys-070816-033654.

[10] Işık M, Bergazin TD, Fox T, Rizzi A, Chodera JD, Mobley DL. Assessing the Accuracy of Octanol-Water Partition Coefficient Predictions in the SAMPL6 Part II Log P Challenge. J Comput Aided Mol Des. 2020 Apr; 34(4):335-370. doi: 10.1007/s10822-020-00295-0.

[11] Laury ML, Wang Z, Gordon AS, Ponder JW. Absolute Binding Free Energies for the SAMPL6 Cucurbit[8]Uril Host-Guest Challenge via the AMOEBA Polarizable Force Field. J Comput Aided Mol Des. 2018 Oct; 32(10):1087-1095. doi: 10.1007/s10822-018-0147-5.

[12] Gapsys V, de Groot BL. Pmx Webserver: A User Friendly Interface for Alchemistry. J Chem Inf Model. 2017 Feb; doi: 10.1021/acs.jcim.6b00498.

[13] Schindler C, Baumann H, Blum A, Böse D, Buchstaller HP, Burgdorf L, Cappel D, Chekler E, Czodrowski P, Dorsch D, Eguida M, Follows B, Fuchß T, Grädler U, Gunera J, Johnson T, Jorand Lebrun C, Karra S, Klein M, Kötzner L, et al. Large-Scale Assessment of Binding Free Energy Calculations in Active Drug Discovery Projects. ChemRxiv. 2020 Jan; doi: 10.26434/chemrxiv.11364884.v1. 
[14] Gapsys V, Pérez-Benito L, Aldeghi M, Seeliger D, van Vlijmen H, Tresadern G, de Groot BL. Large Scale Relative Protein Ligand Binding Affinities Using Non-Equilibrium Alchemy. Chemical Science. 2020; 11(4):1140-1152. doi: 10.1039/C9SC03754C.

[15] Muddana HS, Varnado CD, Bielawski CW, Urbach AR, Isaacs L, Geballe MT, Gilson MK. Blind Prediction of Host-Guest Binding Affinities: A New SAMPL3 Challenge. J Comput Aided Mol Des. 2012 Feb; 26(5):475-487. doi: 10.1007/s10822-012-9554-1.

[16] Skillman AG. SAMPL3: Blinded Prediction of Host-Guest Binding Affinities, Hydration Free Energies, and Trypsin Inhibitors. J Comput Aided Mol Des. 2012 May; 26(5):473-474. doi: 10.1007/s10822-012-9580-z.

[17] Muddana HS, Fenley AT, Mobley DL, Gilson MK. The SAMPL4 Host-Guest Blind Prediction Challenge: An Overview. J Comput Aided Mol Des. 2014 Mar; 28(4):305-317. doi: 10.1007/s10822-014-9735-1.

[18] Peat TS, Dolezal O, Newman J, Mobley DL, Deadman JJ. Interrogating HIV Integrase for Compounds That Bind- a SAMPL Challenge. J Comput Aided Mol Des. 2014 Feb; 28(4):347-362. doi: 10.1007/s10822-014-9721-7.

[19] Gathiaka S, Liu S, Chiu M, Yang H, Stuckey JA, Kang YN, Delproposto J, Kubish G, Dunbar JB, Carlson HA, Burley SK, Walters WP, Amaro RE, Feher VA, Gilson MK. D3R Grand Challenge 2015: Evaluation of Protein-Ligand Pose and Affinity Predictions. J Comput Aided Mol Des. 2016 Sep; 30(9):651-668. doi: 10.1007/s10822-016-9946-8.

[20] Gaieb Z, Liu S, Gathiaka S, Chiu M, Yang H, Shao C, Feher VA, Walters WP, Kuhn B, Rudolph MG, Burley SK, Gilson MK, Amaro RE. D3R Grand Challenge 2: Blind Prediction of Protein-Ligand Poses, Affinity Rankings, and Relative Binding Free Energies. J Comput Aided Mol Des. 2018 Jan; 32(1):1-20. doi: 10.1007/s10822-017-0088-4.

[21] Gaieb Z, Parks CD, Chiu M, Yang H, Shao C, Walters WP, Lambert MH, Nevins N, Bembenek SD, Ameriks MK, Mirzadegan T, Burley SK, Amaro RE, Gilson MK. D3R Grand Challenge 3: Blind Prediction of Protein-Ligand Poses and Affinity Rankings. J Comput Aided Mol Des. 2019 Jan; 33(1):1-18. doi: 10.1007/s10822-018-0180-4.

[22] Parks CD, Gaieb Z, Chiu M, Yang H, Shao C, Walters WP, Jansen JM, McGaughey G, Lewis RA, Bembenek SD, Ameriks MK, Mirzadegan T, Burley SK, Amaro RE, Gilson MK. D3R Grand Challenge 4: Blind Prediction of Protein-Ligand Poses, Affinity Rankings, and Relative Binding Free Energies. J Comput Aided Mol Des. 2020 Feb; 34(2):99-119. doi: 10.1007/s10822-020-00289-y.

[23] Sherborne B, Shanmugasundaram V, Cheng AC, Christ CD, DesJarlais RL, Duca JS, Lewis RA, Loughney DA, Manas ES, McGaughey GB, Peishoff CE, van Vlijmen H. Collaborating to Improve the Use of Free-Energy and Other Quantitative Methods in Drug Discovery. J Comput Aided Mol Des. 2016 Dec; 30(12):1139-1141. doi: 10.1007/s10822-016-9996-y.

[24] Reif MM, Hünenberger PH. Computation of Methodology-Independent Single-Ion Solvation Properties from Molecular Simulations. III. Correction Terms for the Solvation Free Energies, Enthalpies, Entropies, Heat Capacities, Volumes, Compressibilities, and Expansivities of Solvated Ions. J Chem Phys. 2011 Apr; 134(14):144103. doi: 10.1063/1.3567020.

[25] Öhlknecht C, Lier B, Petrov D, Fuchs J, Oostenbrink C. Correcting Electrostatic Artifacts Due to Net-Charge Changes in the Calculation of Ligand Binding Free Energies. Journal of Computational Chemistry. 2020; 41(10):986-999. doi: 10.1002/jcc.26143.

[26] Hünenberger PH, McCammon JA. Ewald Artifacts in Computer Simulations of Ionic Solvation and Ion-Ion Interaction: A Continuum Electrostatics Study. J Chem Phys. 1999 Jan; 110(4):1856-1872. doi: 10.1063/1.477873.

[27] Lin YL, Aleksandrov A, Simonson T, Roux B. An Overview of Electrostatic Free Energy Computations for Solutions and Proteins. J Chem Theory Comput. 2014 Jul; 10(7):2690-2709. doi: 10.1021/ct500195p.

[28] Simonson T, Roux B. Concepts and Protocols for Electrostatic Free Energies. Molecular Simulation. 2016 Sep; 42(13):1090-1101. doi: 10.1080/08927022.2015.1121544.

[29] Ji C, Mei Y. Some Practical Approaches to Treating Electrostatic Polarization of Proteins. Acc Chem Res. 2014 Sep; 47(9):2795-2803. doi: 10.1021/ar500094n.

[30] Zhang C, Lu C, Wang Q, Ponder JW, Ren P. Polarizable Multipole-Based Force Field for Dimethyl and Trimethyl Phosphate. J Chem Theory Comput. 2015 Nov; 11(11):5326-5339. doi: 10.1021/acs.jctc.5b00562.

[31] Kognole AA, Aytenfisu AH, MacKerell AD. Balanced Polarizable Drude Force Field Parameters for Molecular Anions: Phosphates, Sulfates, Sulfamates, and Oxides. J Mol Model. 2020 May; 26(6):152. doi: 10.1007/s00894-020-04399-0.

[32] Cerutti DS, Swope WC, Rice JE, Case DA. Ff14ipq: A Self-Consistent Force Field for Condensed-Phase Simulations of Proteins. J Chem Theory Comput. 2014 Oct; 10(10):4515-4534. doi: 10.1021/ct500643c.

[33] Zhou A, Schauperl M, Nerenberg PS. Benchmarking Electronic Structure Methods for Accurate Fixed-Charge Electrostatic Models. J Chem Inf Model. 2020 Jan; 60(1):249-258. doi: 10.1021/acs.jcim.9b00962.

[34] Schauperl M, Nerenberg PS, Jang H, Wang LP, Bayly Cl, Mobley DL, Gilson MK. Non-Bonded Force Field Model with Advanced Restrained Electrostatic Potential Charges (RESP2). Communications Chemistry. 2020 Apr; 3(1):1-11. doi: 10.1038/s42004-020-0291-4. 
[35] Wang J, Wolf RM, Caldwell JW, Kollman PA, Case DA. Development and Testing of a General Amber Force Field. J Comput Chem. 2004 Jul; 25(9):1157-1174. doi: 10.1002/jcc.20035.

[36] Wang J, Wang W, Kollman PA, Case DA. Automatic Atom Type and Bond Type Perception in Molecular Mechanical Calculations. J Mol Graph Model. 2006 Oct; 25(2):247-260. doi: 10.1016/j.jmgm.2005.12.005.

[37] Mobley DL, Bannan CC, Rizzi A, Bayly Cl, Chodera JD, Lim VT, Lim NM, Beauchamp KA, Slochower DR, Shirts MR, Gilson MK, Eastman PK. Escaping Atom Types in Force Fields Using Direct Chemical Perception. J Chem Theory Comput. 2018 Oct; doi: 10.1021/acs.jctc.8b00640.

[38] Vanommeslaeghe K, Hatcher E, Acharya C, Kundu S, Zhong S, Shim J, Darian E, Guvench O, Lopes P, Vorobyov I, Mackerell AD. CHARMM General Force Field: A Force Field for Drug-like Molecules Compatible with the CHARMM All-Atom Additive Biological Force Fields. Journal of Computational Chemistry. 2009; p. NA-NA. doi: 10.1002/jcc.21367.

[39] Vanommeslaeghe K, MacKerell AD. Automation of the CHARMM General Force Field (CGenFF) I: Bond Perception and Atom Typing. J Chem Inf Model. 2012 Dec; 52(12):3144-3154. doi: 10.1021/ci300363c.

[40] Vanommeslaeghe K, Raman EP, MacKerell AD. Automation of the CHARMM General Force Field (CGenFF) II: Assignment of Bonded Parameters and Partial Atomic Charges. J Chem Inf Model. 2012 Dec; 52(12):3155-3168. doi: 10.1021/ci3003649.

[41] Kaminski GA, Friesner RA, Tirado-Rives J, Jorgensen WL. Evaluation and Reparametrization of the OPLS-AA Force Field for Proteins via Comparison with Accurate Quantum Chemical Calculations on Peptides. J Phys Chem B. 2001 Jul; 105(28):6474-6487. doi: 10.1021/jp003919d.

[42] Harder E, Damm W, Maple J, Wu C, Reboul M, Xiang JY, Wang L, Lupyan D, Dahlgren MK, Knight JL, Kaus JW, Cerutti DS, Krilov G, Jorgensen WL, Abel R, Friesner RA. OPLS3: A Force Field Providing Broad Coverage of Drug-like Small Molecules and Proteins. J Chem Theory Comput. 2016 Jan; 12(1):281-296. doi: 10.1021/acs.jctc.5b00864.

[43] Mobley DL, Amezcua M, Ponder J, Khalak Y, Yigitkan Eken E, Almeida N, Isaacs L, Gibb B, Kellett K, Serrilon D, The SAMPL7 Host-Guest Challenge Virtual Workshop. Zenodo; 2020. doi: 10.5281/zenodo.3674155.

[44] Saric D, Kohns M, Vrabec J. Dielectric Constant and Density of Aqueous Alkali Halide Solutions by Molecular Dynamics: A Force Field Assessment. J Chem Phys. 2020 Apr; 152(16):164502. doi: 10.1063/1.5144991.

[45] Vega C. Water: One Molecule, Two Surfaces, One Mistake. Molecular Physics. 2015 May; 113(9-10):1145-1163. doi: 10.1080/00268976.2015.1005191.

[46] González MA. Force Fields and Molecular Dynamics Simulations. JDN. 2011; 12:169-200. doi: 10.1051/sfn/201112009.

[47] Guillot B. A Reappraisal of What We Have Learnt during Three Decades of Computer Simulations on Water. Journal of Molecular Liquids. 2002 Nov; 101(1):219-260. doi: 10.1016/S0167-7322(02)00094-6.

[48] Henriksen NM, Gilson MK. Evaluating Force Field Performance in Thermodynamic Calculations of Cyclodextrin Host-Guest Binding: Water Models, Partial Charges, and Host Force Field Parameters. J Chem Theory Comput. 2017 Sep; 13(9):4253-4269. doi: 10.1021/acs.jctc.7b00359.

[49] Yin J, Henriksen NM, Muddana HS, Gilson MK. Bind3P: Optimization of a Water Model Based on Host-Guest Binding Data. J Chem Theory Comput. 2018 Jul; 14(7):3621-3632. doi: 10.1021/acs.jctc.8b00318.

[50] Gibb CLD, Gibb BC. Anion Binding to Hydrophobic Concavity Is Central to the Salting-in Effects of Hofmeister Chaotropes. J Am Chem Soc. 2011 May; 133(19):7344-7347. doi: 10.1021/ja202308n.

[51] Thormann E. On Understanding of the Hofmeister Effect: How Addition of Salt Alters the Stability of Temperature Responsive Polymers in Aqueous Solutions | Request PDF. RSC Adv. 2012 Jul; p. 8297-8305. doi: DOI: 10.1039/c2ra20164j.

[52] Gao K, Yin J, Henriksen NM, Fenley AT, Gilson MK. Binding Enthalpy Calculations for a Neutral Host-Guest Pair Yield Widely Divergent Salt Effects across Water Models. J Chem Theory Comput. 2015 Oct; 11(10):4555-4564. doi: 10.1021/acs.jctc.5b00676.

[53] Carnegie RS, Gibb CLD, Gibb BC. Anion Complexation and The Hofmeister Effect. Angew Chem. 2014 Oct; 126(43):11682-11684. doi: 10.1002/ange.201405796.

[54] Gibb CLD, Gibb BC. Well-Defined, Organic Nanoenvironments in Water: The Hydrophobic Effect Drives a Capsular Assembly. J Am Chem Soc. 2004 Sep; 126(37):11408-11409. doi: 10.1021/ja0475611.

[55] Saltzman A, Tang D, Gibb BC, Ashbaugh HS. Emergence of Non-Monotonic Deep Cavity Cavitand Assembly with Increasing Portal Methylation. Mol Syst Des Eng. 2020 Mar; 5(3):656-665. doi: 10.1039/C9ME00076C.

[56] Brown A. Analysis of Cooperativity by Isothermal Titration Calorimetry. Int J Mol Sci. 2009 Aug; 10(8):3457-3477. doi: 10.3390/ijms10083457. 
[57] Ma YL, Ke H, Valkonen A, Rissanen K, Jiang W. Achieving Strong Positive Cooperativity through Activating Weak Non-Covalent Interactions. Angewandte Chemie International Edition. 2018; 57(3):709-713. doi: 10.1002/anie.201711077.

[58] Ndendjio SZ, Liu W, Yvanez N, Meng Z, Zavalij PY, Isaacs L. Triptycene Walled Glycoluril Trimer: Synthesis and Recognition Properties. New J Chem. 2019 Dec; 44(2):338-345. doi: 10.1039/C9NJ05336K.

[59] Suating P, T Nguyen T, E Ernst N, Wang Y, H Jordan J, D Gibb CL, S Ashbaugh H, C Gibb B. Proximal Charge Effects on Guest Binding to a Non-Polar Pocket. Chemical Science. 2020; 11(14):3656-3663. doi: 10.1039/C9SC06268H.

[60] Kellett K, Slochower D, Schauperl M, Duggan BM, Gilson M. Experimental Characterization of the Association of Nine Novel Cyclodextrin Derivatives with Two Guest Compounds. chemRxiv. 2020 Jul; doi: 10.26434/chemrxiv.12663065.v1.

[61] Lee J, Tofoleanu F, Pickard FC, König G, Huang J, Damjanović A, Baek M, Seok C, Brooks BR. Absolute Binding Free Energy Calculations of CBClip Host-Guest Systems in the SAMPL5 Blind Challenge. J Comput Aided Mol Des. 2017 Jan; 31(1):71-85. doi: 10.1007/s10822-016-99682.

[62] Ma D, Zavalij PY, Isaacs L. Acyclic Cucurbit[n]Uril Congeners Are High Affinity Hosts. J Org Chem. 2010 Jul; 75(14):4786-4795. doi: $10.1021 /$ jo100760g.

[63] Biedermann F, Rauwald U, Cziferszky M, Williams KA, Gann LD, Guo BY, Urbach AR, Bielawski CW, Scherman OA. Benzobis(Imidazolium)-Cucurbit[8]Uril Complexes for Binding and Sensing Aromatic Compounds in Aqueous Solution. Chemistry - A European Journal. 2010 Dec; 16(46):13716-13722. doi: 10.1002/chem.201002274.

[64] Gallicchio E, Levy RM. Prediction of SAMPL3 Host-Guest Affinities with the Binding Energy Distribution Analysis Method (BEDAM). J Comput Aided Mol Des. 2012 May; 26(5):505-516. doi: 10.1007/s10822-012-9552-3.

[65] Naïm M, Bhat S, Rankin KN, Dennis S, Chowdhury SF, Siddiqi I, Drabik P, Sulea T, Bayly Cl, Jakalian A, Purisima EO. Solvated Interaction Energy (SIE) for Scoring Protein-Ligand Binding Affinities. 1. Exploring the Parameter Space. J Chem Inf Model. 2007 Jan; 47(1):122-133. doi: $10.1021 / c i 600406 v$

[66] Yin J, Henriksen NM, Slochower DR, Shirts MR, Chiu MW, Mobley DL, Gilson MK. Overview of the SAMPL5 Host-Guest Challenge: Are We Doing Better? J Comput Aided Mol Des. 2017; 31(1):1-19. doi: 10.1007/s10822-016-9974-4.

[67] Liu W, Lu X, Xue W, Samanta SK, Zavalij PY, Meng Z, Isaacs L. Hybrid Molecular Container Based on Glycoluril and Triptycene: Synthesis, Binding Properties, and Triggered Release. Chem Eur J. 2018 Sep; 24(53):14101-14110. doi: 10.1002/chem.201802981.

[68] Ndendjio SAZ, Isaacs L. Molecular Recognition Properties of Acyclic Cucurbiturils toward Amino Acids, Peptides, and a Protein. Supramolecular Chemistry. 2019 Jul; 31(7):432-441. doi: 10.1080/10610278.2019.1619737.

[69] Biedermann F, Uzunova VD, Scherman OA, Nau WM, De Simone A. Release of High-Energy Water as an Essential Driving Force for the High-Affinity Binding of Cucurbit[n]Urils. J Am Chem Soc. 2012 Sep; 134(37):15318-15323. doi: 10.1021/ja303309e.

[70] Monroe JI, Shirts MR. Converging Free Energies of Binding in Cucurbit[7]Uril and Octa-Acid Host-Guest Systems from SAMPL4 Using Expanded Ensemble Simulations. J Comput Aided Mol Des. 2014 Apr; 28(4):401-415. doi: 10.1007/s10822-014-9716-4.

[71] Liu W, Lu X, Meng Z, Isaacs L. A Glycoluril Dimer-Triptycene Hybrid Receptor: Synthesis and Molecular Recognition Properties. Org Biomol Chem. 2018; 16(35):6499-6506. doi: 10.1039/C8OB01575A.

[72] Barnett JW, Sullivan MR, Long JA, Tang D, Nguyen T, Ben-Amotz D, Gibb BC, Ashbaugh HS. Spontaneous Drying of Non-Polar Deep-Cavity Cavitand Pockets in Aqueous Solution. Nature Chemistry. 2020 May; p. 1-6. doi: 10.1038/s41557-020-0458-8.

[73] Gibb CLD, Gibb BC. Guests of Differing Polarities Provide Insight into Structural Requirements for Templates of Water-Soluble NanoCapsules. Tetrahedron. 2009 Aug; 65(35):7240-7248. doi: 10.1016/j.tet.2009.01.106.

[74] Gibb CLD, Gibb BC. Binding of Cyclic Carboxylates to Octa-Acid Deep-Cavity Cavitand. J Comput Aided Mol Des. 2014 Apr; 28(4):319-325. doi: 10.1007/s10822-013-9690-2.

[75] Ewell J, Gibb BC, Rick SW. Water Inside a Hydrophobic Cavitand Molecule. J Phys Chem B. 2008 Aug; 112(33):10272-10279. doi: $10.1021 /$ jp804429n.

[76] Kellett K, Kantonen SA, Duggan BM, Gilson MK. Toward Expanded Diversity of Host-Guest Interactions via Synthesis and Characterization of Cyclodextrin Derivatives. J Solution Chem. 2018 Nov; 47(10):1597-1608. doi: 10.1007/s10953-018-0769-1.

[77] Slochower DR, Henriksen NM, Wang LP, Chodera JD, Mobley DL, Gilson MK. Binding Thermodynamics of Host-Guest Systems with SMIRNOFF99Frosst 1.0.5 from the Open Force Field Initiative. J Chem Theory Comput. 2019 Nov; 15(11):6225-6242. doi: 10.1021 /acs.jctc.9b00748. 
[78] Carrazana J, Jover A, Meijide F, Soto VH, Vázquez Tato J. Complexation of Adamantyl Compounds by $\beta$-Cyclodextrin and Monoaminoderivatives. J Phys Chem B. 2005 May; 109(19):9719-9726. doi: 10.1021/jp0505781.

[79] Rizzi A, Grinaway P, Parton D, Shirts M, Wang K, Eastman P, Friedrichs M, Pande V, Branson K, Mobley D, Chodera J. YANK: A GPU-Accelerated Platform for Alchemical Free Energy Calculations. .; .

[80] Wang K, Chodera JD, Yang Y, Shirts MR. Identifying Ligand Binding Sites and Poses Using GPU-Accelerated Hamiltonian Replica Exchange Molecular Dynamics. J Comput Aided Mol Des. 2013 Dec; 27(12):989-1007. doi: 10.1007/s10822-013-9689-8.

[81] Friedrichs MS, Eastman P, Vaidyanathan V, Houston M, Legrand S, Beberg AL, Ensign DL, Bruns CM, Pande VS. Accelerating Molecular Dynamic Simulation on Graphics Processing Units. Journal of Computational Chemistry. 2009; 30(6):864-872. doi: 10.1002/jcc.21209.

[82] Eastman P, Pande V. OpenMM: A Hardware-Independent Framework for Molecular Simulations. Computing in Science Engineering. 2010 Jul; 12(4):34-39. doi: 10.1109/MCSE.2010.27.

[83] Eastman P, Pande VS. Constant Constraint Matrix Approximation: A Robust, Parallelizable Constraint Method for Molecular Simulations. J Chem Theory Comput. 2010 Feb; 6(2):434-437. doi: 10.1021/ct900463w.

[84] Eastman P, Pande VS. Efficient Nonbonded Interactions for Molecular Dynamics on a Graphics Processing Unit. Journal of Computational Chemistry. 2010; 31(6):1268-1272. doi: 10.1002/jcc.21413.

[85] Eastman P, Friedrichs MS, Chodera JD, Radmer RJ, Bruns CM, Ku JP, Beauchamp KA, Lane TJ, Wang LP, Shukla D, Tye T, Houston M, Stich T, Klein C, Shirts MR, Pande VS. OpenMM 4: A Reusable, Extensible, Hardware Independent Library for High Performance Molecular Simulation. J Chem Theory Comput. 2013 Jan; 9(1):461-469. doi: 10.1021/ct300857j.

[86] Shirts MR, Chodera JD. Statistically Optimal Analysis of Samples from Multiple Equilibrium States. J Chem Phys. 2008 Sep; $129(12): 124105$. doi: 10.1063/1.2978177.

[87] Rizzi A, Murkli S, McNeill JN, Yao W, Sullivan M, Gilson MK, Chiu MW, Isaacs L, Gibb BC, Mobley DL, Chodera JD. Overview of the SAMPL6 Host-Guest Binding Affinity Prediction Challenge. Journal of Computer-Aided Molecular Design. 2018 Oct; 32(10):937-963. doi: 10.1007/s10822-018-0170-6.

[88] Procacci P. Precision and Computational Efficiency of Nonequilibrium Alchemical Methods for Computing Free Energies of Solvation. II. Unidirectional Estimates. J Chem Phys. 2019 Oct; 151(14):144115. doi: 10.1063/1.5120616.

[89] Izadi S, Onufriev AV. Accuracy Limit of Rigid 3-Point Water Models. J Chem Phys. 2016 Aug; 145(7). doi: 10.1063/1.4960175.

[90] Marenich AV, Cramer C], Truhlar DG. Universal Solvation Model Based on Solute Electron Density and on a Continuum Model of the Solvent Defined by the Bulk Dielectric Constant and Atomic Surface Tensions. J Phys Chem B. 2009 May; 113(18):6378-6396. doi: $10.1021 /$ jp810292n. 
${ }_{1084} 13$ Supplementary Information

1085 An archive copy of SAMPL7 GitHub repository host-guest challenge directory. 